\title{
Planetary nebulae near the Galactic center: Identifications ${ }^{\star}$
}

\author{
G. H. Jacoby ${ }^{1, \star \star, \star \star \star}$ and G. Van de Steene ${ }^{2}$ \\ 1 WIYN Observatory, PO Box 26732, Tucson, AZ, 85726, USA \\ ${ }^{2}$ Royal Observatory of Belgium, Ringlaan 3, 1180 Brussels, Belgium
}

Received 5 December 2003 / Accepted 3 February 2004

\begin{abstract}
We surveyed the central $4 \times 4$ degrees of the Galactic center for planetary nebulae in the light of [S III] $\lambda 9532$ and found $94 \mathrm{PNe}$ that were not previously known, plus 3 that were previously identified as possible candidates. For 63 of these 97 objects, we obtained spectra that are consistent with highly reddened PN while the other 34 could not be recovered spectroscopically and remain unverified. Of the 94 candidates, 54 and 57 were detected via radio at 3 and $6 \mathrm{~cm}$, respectively. An additional 20 PNe candidates were found during follow-up $\mathrm{H} \alpha$ imaging but have not yet been verified spectroscopically. Based on the properties of IRAS sources in this region of the Galaxy, and on the total luminosity of the Galactic bulge, the expected number of PNe is $\sim 250$, only $50 \%$ more than the 160 PNe candidates now known. Thus, surveys for PNe in the bulge are approximately two-thirds complete with the remainder likely hidden behind dust.
\end{abstract}

Key words. ISM: planetary nebulae: general - Galaxy: bulge - methods: observational

\section{Introduction and motivation}

Estimates of the number of planetary nebulae $(\mathrm{PNe})$ in the Galaxy have always been subject to large uncertainties, ranging, for example, from 6000 to 80000 (Peimbert 1993). The principal obstacle in deriving an accurate count is the very high level of extinction close to the galactic plane, especially when looking toward the Galactic center where a large fraction of the population is expected. Interest in the population count stems from studies of the chemical enrichment rates (e.g., Peimbert 1987) and comparisons between the populations in our Galaxy and those in external systems (e.g., Jacoby 1980; Peimbert 1993).

For example, when looking at M 31, the PN system is strongly concentrated toward the nucleus (Ciardullo et al. 1989). That is, the PN density follows the radial increase of starlight inward. Yet, the Galactic distribution fails to rise accordingly, in contrast to the rapidly increasing population of IRAS sources and OH/IR stars that have IR colors of PNe (see Fig. 2 of Pottasch et al. 1988). If, in fact, the PNe were to follow

Send offprint requests to: G. H. Jacoby, e-mail: gjacoby@wiyn.org

* Observations made with the Burrell Schmidt of the Warner and Swasey Observatory, Case Western Reserve University.

$\star \star$ Visiting Astronomer, Kitt Peak National Observatory, National Optical Astronomy Observatory, which is operated by the Association of Universities for Research in Astronomy, Inc., under cooperative agreement with the National Science Foundation.

$\star \star \star$ Visiting Astronomer, Cerro Tololo Interamerican Observatory, National Optical Astronomy Observatory, which is operated by the Association of Universities for Research in Astronomy, Inc., under cooperative agreement with the National Science Foundation. the same distribution as these sources, then a relative lifetime argument implies that there are $\sim 320$ PNe within 2 degrees of the Galactic center (see Fig. 3 of Pottasch et al. 1988). Yet, prior to this survey, initiated in 1994 , only $34 \mathrm{PNe}$, or $10 \%$ of the expected number, were known in this region.

The expectation of $320 \mathrm{PNe}$, though, is highly approximate because not all color-selected IRAS candidates are true PNe. Van de Steene \& Pottasch (1995) found that roughly $25 \%$ of their IRAS sources could be recovered in a radio survey, implying a minimum estimate of $80 \mathrm{PNe}$ for the central 2 degrees. On the other hand, many PNe are not detectable by IRAS and so, IRAS counts underestimate the true number of PNe. Unfortunately, there is no reliable estimate for this factor because neither the IRAS survey nor PN surveys are complete. We return to this question in Sect. 3 where we derive another estimate for the number of $\mathrm{PNe}$ in the Galactic bulge.

Beyond the simple counting statistics of PNe, the greater value of their study in and near the Galactic center is to use them as probes of the kinematic and chemical history of the bulge population of stars. If it were possible to identify many hundreds of PNe in the bulge region, one could map out the matter distribution, including any dark matter contributions, as was done in the galaxy NGC 5128 by Hui et al. (1995).

Perhaps most importantly, follow-up spectroscopy of PNe can tell us the rate at which the alpha elements were enhanced near the Galactic center. In particular, the elements $\mathrm{O}, \mathrm{Ne}, \mathrm{S}$, and Ar survive the stellar evolution and nucleosynthesis process unaffected from when the star was formed (Forestini \& Charbonnel 1997). By analyzing a PN spectrum, one can deduce the time of formation of the progenitor star, as well as its 
initial chemical composition. By analyzing many PNe, a timeline for elemental enhancements can be constructed, as was done for the LMC by Dopita et al. (1997). Once the chemical compositions are known for many PNe, they can complement the stellar compositions (McWilliam \& Rich 1994) by providing information on different elements; or, $\mathrm{PNe}$ can be used in place of the more observationally challenging stellar composition analysis.

For these reasons, the interest in Galactic center PNe has grown since we began our survey. The most productive general survey for Galactic PNe has been described by Parker \& Phillipps (1998) and Parker et al. (2003) netting 1214 newly identified $\mathrm{PNe}$ in $\mathrm{H} \alpha$. Searches for PNe specifically toward the Galactic center and bulge have also been carried out by Gómez et al. (1997), Beaulieu et al. (1998), Kohoutek (2002), and Boumis et al. (2003).

In this paper, we report on the identification of many additional PNe within a few degrees of the Galactic center that were not previously known, thanks to a wide-field near-IR survey initially motivated by discussions with Stuart Pottasch. Van de Steene \& Jacoby (2001) have already reported the results of synthesis radio observations at 3 and $6 \mathrm{~cm}$ that were carried out at the Australian Telescope Compact Array to improve the determination of extinction measures for these highly obscured PNe. In a subsequent paper, we will report the results of our spectroscopic follow-up survey that provides the validation for most of these objects as $\mathrm{PNe}$, as well as radial velocities for kinematic studies, and in a few cases, chemical composition estimates and ages for the $\mathrm{PNe}$ and their progenitors to explore the chemical enrichment history of the Galactic bulge.

\section{Observational approach}

The success of this survey was made possible by the novel use of narrow-band imaging in the near-IR [SIII] line at $9532 \AA$. The [OIII] line at $5007 \AA$ is more commonly used for surveys because of its extreme intrinsic brightness. The [SIII] line, though, has the attactive property of being the apparently brightest line in the spectra of typical PNe when the $V$-band extinction is between 4 and $12 \mathrm{mag}$. Beyond 12 mag of extinction, $\mathrm{Br} \gamma$ becomes the survey line of choice from the ground.

As will be shown in a later paper where we present spectra of the new PNe, the selection of the [SIII] line was nearly optimal. We will show that using the [OIII] $\lambda 5007$ line would have been disasterous due to the effects of reddening. It is much less obvious, however, that $\mathrm{H} \alpha$ would have been a poorer choice. In fact, as we show below, $\mathrm{H} \alpha$ offers some advantages. As a general rule, though, $\mathrm{H} \alpha$ surveys for PNe suffer badly from confusion with emission-line stars and HII regions and cannot be used reliably unless another filter centered on a high excitation ion (e.g., [SIII]) is used in conjunction.

We carried out the survey of the central $4 \times 4$ degrees using the Case Western Reserve University (CWRU) Burrell Schmidt telescope on Kitt Peak in August 1994 and July 1995. At that time this $0.6-\mathrm{m}$ telescope had a uniquely wide $69^{\prime} \times 69^{\prime}$ field of view with a $2 \mathrm{~K} \times 2 \mathrm{~K}$ STIS CCD. The CCD's $21 \mu \mathrm{m}$ pixels subtend $2.028^{\prime \prime}$ on the sky, which is relatively coarse sampling that limits the accuracy of any photometry or the identification
Table 1. Field centers for the near-IR PN survey.

\begin{tabular}{lccc}
\hline \hline Field & $\alpha(2000)$ & $\delta(2000)$ & UT Date \\
\hline 1 & 173637 & -292327 & 14 July 1994 \\
2 & 173857 & -283519 & 14 July 1994 \\
3 & 174114 & -274702 & 14 July 1994 \\
4 & 174329 & -265835 & 15 July 1994 \\
5 & 174019 & -295354 & 15 July 1994 \\
6 & 174237 & -290531 & 15 July 1994 \\
7 & 174454 & -281659 & 15 July 1994 \\
8 & 174708 & -272818 & 19 June 1995 \\
9 & 174403 & -302357 & 19 June 1995 \\
10 & 174620 & -293520 & 19 June 1995 \\
11 & 174835 & -284634 & 19 June 1995 \\
12 & 175049 & -275740 & 20 June 1995 \\
13 & 174749 & -305337 & 20 June 1995 \\
14 & 175005 & -300445 & 20 June 1995 \\
15 & 175219 & -291546 & 20 June 1995 \\
16 & 175431 & -282639 & 21 June 1995 \\
\hline
\end{tabular}

of stellar sources. Many of the Galactic center PNe, though, are slightly extended ( 1 " at the Galactic center corresponds to a radius of $0.019 \mathrm{pc}$ ), and so, this deficiency was not serious. In addition to its wide field, this CCD was unthinned (i.e., it is a "thick" CCD), and this characteristic proved highly advantageous for working in the near-IR. With the usually more desirable thinned CCDs, fringing at the [SIII] wavelength is extremely severe and flat-fielding the images is impossible. Thus, despite the small size of the telescope and the observational challenge of working from a northern site, the problem of finding PNe in the Galactic bulge actually was extremely wellsuited to the equipment at hand.

For the on-band [SIII] images, a special filter was purchased having a central wavelength of $9535 \AA$ and bandpass $(F W H M)$ of $29 \AA$ in the $\mathrm{f} / 3.5$ beam of the CWRU Schmidt. We used a broad RG-850 to serve as an "off-band" filter.

\subsection{The [SIII] survey}

The coordinates for the centers of the 16 [SIII] survey fields and their dates of observations are given in Table 1. Exposure times were $40 \mathrm{~min}$ for the [SIII] on-band, split into two 20-min exposures to provide cosmic ray rejection, and 2 min for the "off-band" RG-850 exposures, also split into two exposures. The sky was never reliably photometric during the survey, and so we cannot report fluxes for the nebulae at [SIII].

All images were reduced in the usual manner with the CCDPROC task in the IRAF package using twilight sky images for flat-fielding. The [SIII] and RG-850 images were aligned, and the RG-850 image was scaled and subtracted from the [SIII] image. The resulting difference image was visually scanned for positive residuals that signal a [SIII] emission-line candidate. Coordinates for the candidates were derived using the IRAF FINDER package, referencing the coordinate system to the HST Guide Star Catalog. Due to the coarse pixel sampling and the extended nature of the objects, the derived coordinates have accuracies limited to $\sim 5^{\prime \prime}$. The list of candidates is given in Table 2, along with their diameters, their 
Table 2. Properties of new Galactic bulge PNe.

\begin{tabular}{|c|c|c|c|c|c|c|c|}
\hline $\mathrm{JaSt}$ & RA (J2000.0) & Dec (J2000) & $\begin{array}{c}\operatorname{Diam}^{a} \\
(\operatorname{arcsec})\end{array}$ & $\begin{array}{l}\log \text { Flux }^{b} \\
\mathrm{H} \alpha+[\mathrm{NII}]\end{array}$ & $\begin{array}{c}\text { Flux ratio }^{c} \\
\mathrm{H} \alpha / \mathrm{H} \alpha+[\mathrm{NII}]\end{array}$ & $\begin{array}{c}\begin{array}{c}6 \mathrm{~cm} \mathrm{Flux}^{d} \\
(\mathrm{mJy})\end{array}\end{array}$ & Comment $^{e}$ \\
\hline 1 & 173443.64 & -294705.03 & 5 & -13.98: & 0.532 & 1.6 & \\
\hline 2 & 173500.96 & -292215.72 & 5 & -13.22 & 0.883 & 4.3 & \\
\hline 3 & 173522.90 & -292217.58 & 7 & -13.08 & 0.927 & 12.4 & \\
\hline 4 & 173537.47 & -291317.67 & 8 & -13.17 & 0.889 & 4.1 & \\
\hline 5 & 173552.51 & -285827.95 & 4 & -13.11 & 0.850 & 12.7 & \\
\hline 6 & 173638.71 & -291722.05 & - & 0.00 & & & \\
\hline 7 & 173826.69 & -284706.48 & 13 & -13.41 & 0.246 & & \\
\hline 8 & 173827.74 & -285201.31 & 8 & -13.39 & 0.895 & 3.8 & \\
\hline 9 & 173845.64 & -290859.27 & 9 & -13.83 & 0.426 & 4.0 & \\
\hline 10 & 173847.51 & -290115.17 & 5 & 0.00 & & & \\
\hline 11 & 173900.55 & -301135.23 & 16 & $-14.62:$ & 0.36 : & 3.9 & \\
\hline 12 & 173859.28 & -284641.87 & 2 & $-14.09:$ & & & \\
\hline 13 & 173901.80 & -293858.07 & - & & & & \\
\hline 14 & 173912.74 & -291410.74 & - & & & & \\
\hline 15 & 173917.11 & -290136.24 & - & & & & \\
\hline 16 & 173922.70 & -294146.08 & 4 & -13.83 & 0.665 & 27.0 & \\
\hline 17 & 173931.32 & -272746.78 & 8 & -12.59 & 0.702 & 10.0 & K6-7 \\
\hline 18 & 173934.42 & -290835.39 & - & & & & \\
\hline 19 & 173939.38 & -274722.58 & 6 & -12.84 & 0.965 & 6.8 & K6-8 \\
\hline 20 & 173950.59 & -290448.36 & 5 & 0.00 & & & \\
\hline 21 & 173952.92 & -274420.54 & 21 & $-13.22:$ & 0.701 & & \\
\hline 22 & 174011.98 & -302915.65 & - & & & & \\
\hline 23 & 174023.17 & -274912.04 & 3 & -13.49 & 0.500 & 3.7 & \\
\hline 24 & 174028.23 & -301351.30 & - & 0.00 & - & 16.0 & \\
\hline 25 & 174027.83 & -293006.05 & - & 0.00 & & & \\
\hline 26 & 174033.52 & -294614.98 & 11 & 0.00 & 0.745 & 14.1 & \\
\hline 27 & 174042.34 & -281231.90 & 5 & -13.82 & 1.000 & & \\
\hline 29 & 174101.64 & -285810.87 & - & 0.00 & & & \\
\hline 30 & 174102.27 & -284626.68 & - & 0.00 & & & \\
\hline 31 & 174127.93 & -285251.61 & 7 & $-14.49:$ & 0.790: & 11.5 & \\
\hline 32 & 174143.91 & -273352.32 & 6 & 0.00 & & & \\
\hline 33 & 174155.57 & -293728.89 & 5 & & & & \\
\hline 34 & 174154.80 & -270320.33 & 6 & -13.78 & 0.960 & 1.7 & \\
\hline 35 & 174208.71 & -285537.05 & 28 & & & & \\
\hline 36 & 174225.20 & -275536.36 & 5 & -13.03 & 0.872 & 31.1 & \\
\hline 37 & 174228.60 & -300934.93 & 10 & 0.00 & - & 13.5 & IRAS $17392-3008$ \\
\hline 38 & 174232.41 & -273315.18 & 8 & $-13.06:$ & 0.449 & 2.8 & \\
\hline 39 & 174245.14 & -262920.34 & - & 0.00 & & & \\
\hline 40 & 174250.16 & -284414.69 & 7 & 0.00 & & & \\
\hline 41 & 174249.96 & -272119.68 & 7 & -12.73 & 0.906 & 16.7 & \\
\hline 42 & 174317.06 & -264417.67 & 7 & -12.81 & 0.878 & 12.9 & K6-10 \\
\hline 43 & 174321.78 & -284518.68 & - & 0.00 & & & \\
\hline 44 & 174323.48 & -273406.03 & 7 & -13.04 & 0.649 & 3.8: & \\
\hline 45 & 174323.44 & -271116.91 & 33 & $-12.85:$ & 0.185 & & \\
\hline 46 & 174330.43 & -264732.33 & 6 & -12.29 & 0.564 & 20.8 & \\
\hline 47 & 174333.61 & -281845.49 & 15 & & & & \\
\hline 48 & 174359.26 & -274432.56 & - & & & & \\
\hline 49 & 174404.34 & -281557.86 & 6 & & - & 8.2 & \\
\hline 50 & 174433.97 & -262457.10 & 15 & & & & \\
\hline 51 & 174440.12 & -292224.31 & - & 0.00 & & & \\
\hline 52 & 174437.30 & -264725.23 & 5 & -12.47 & 0.665 & 24.0 & \\
\hline 53 & 174439.78 & -265615.66 & 6 & 0.00 & & & \\
\hline 54 & 174511.06 & -273236.80 & 15 & 0.00 & - & 82.3 & IRAS 17420-2731 \\
\hline
\end{tabular}


Table 2. continued.

\begin{tabular}{|c|c|c|c|c|c|c|c|}
\hline JaSt & RA (J2000.0) & Dec (J2000) & $\begin{array}{l}\text { Diam }^{a} \\
(\operatorname{arcsec})\end{array}$ & $\begin{array}{l}\log _{\text {Flux }}^{b} \\
\mathrm{H} \alpha+[\mathrm{NII}]\end{array}$ & $\begin{array}{c}\text { Flux ratio }^{c} \\
\mathrm{H} \alpha / \mathrm{H} \alpha+[\mathrm{NII}]\end{array}$ & $\begin{array}{c}6 \text { cm Flux }^{d} \\
(\mathrm{mJy})\end{array}$ & Comment $^{e}$ \\
\hline 55 & 174537.36 & -270118.44 & 10 & -13.18 & 0.485 & 20.0 & \\
\hline 56 & 174547.05 & -273042.03 & 6 & 0.00 & 0.613 & 14.1 & \\
\hline 57 & 174630.97 & -301652.68 & - & & & & \\
\hline 58 & 174652.20 & -303742.83 & 14 & $-14.26:$ & 0.469 & 29.5 & \\
\hline 59 & 174709.66 & -283116.42 & 9 & 0.00 & & & \\
\hline 60 & 174753.91 & -293649.67 & 8 & 0.00 & - & 6.5 & IRAS $17420-2731$ \\
\hline 61 & 174823.02 & -294044.65 & 7 & 0.00 & & & \\
\hline 62 & 174846.10 & -275738.61 & 6 & 0.00 & & & \\
\hline 63 & 174846.27 & -272537.22 & 10 & -13.37 & 0.261 & 3.9 & \\
\hline 64 & 174856.04 & -310641.95 & 5 & -13.00 & 0.841 & 28.3 & \\
\hline 65 & 174920.02 & -303605.57 & 5 & -12.66 & 0.941 & & IRAS 17461-3035 \\
\hline 66 & 174922.15 & -295927.02 & 5 & -12.86 & 0.902 & 65.3 & \\
\hline 67 & 174928.10 & -292047.56 & - & & 1.00: & 27.8 & \\
\hline 68 & 174950.87 & -300310.47 & 5 & -13.96 & 0.953 & 5.9 & \\
\hline 69 & 175010.04 & -291905.14 & 14 & -12.81 & 0.380 & 4.0: & \\
\hline 70 & 175021.07 & -283902.46 & 11 & 0.00 & - & 13.0 & ?IRAS 17471-2838 \\
\hline 71 & 175023.32 & -283310.95 & 5 & -13.74 & 0.577 & 34.0 & \\
\hline 72 & 175029.06 & -300341.75 & 22 & $-13.33:$ & & & \\
\hline 73 & 175047.74 & -295316.01 & 7 & -12.49 & 0.648 & 13.7 & IRAS $17475-2952$ \\
\hline 74 & 175046.85 & -284435.42 & 5 & $-13.90:$ & 0.611 & 22.7 & \\
\hline 75 & 175048.08 & -292443.60 & 5 & -13.21 & 0.692 & 22.5 & IRAS 17476-292 \\
\hline 76 & 175056.47 & -283124.63 & - & & 0.732 & 7.4 & \\
\hline 77 & 175111.65 & -285627.20 & 10 & -12.56 & 0.922 & 49.0 & ?IRAS 17480-2855 \\
\hline 78 & 175124.68 & -283540.34 & 11 & & 0.870 & 12.3 & \\
\hline 79 & 175153.63 & -293053.41 & 5 & -12.54 & 0.545 & 4.1 & \\
\hline 80 & 175155.54 & -274802.46 & 25 & $-12.46:$ & 0.239 & & \\
\hline 81 & 175204.35 & -273639.28 & - & 0.00 & $0.135:$ & 20.8 & IRAS $17480-2855$ \\
\hline 82 & 175205.08 & -280549.67 & 12 & & & & \\
\hline 83 & 175245.17 & -295105.21 & - & & 0.673 & 4.0 & \\
\hline 84 & 175246.17 & -284838.80 & 20 & $-13.35:$ & & & \\
\hline 85 & 175249.05 & -294154.92 & 15 & -12.26 & 0.614 & 5.3 & \\
\hline 86 & 175252.20 & -293000.07 & 7 & -12.80 & 0.865 & 8.5 & \\
\hline 87 & 175300.28 & -284415.50 & 15 & $-13.45:$ & & & \\
\hline 88 & 175300.89 & -290544.08 & 14 & $-12.85:$ & 0.735 & & \\
\hline 89 & 175306.73 & -281809.95 & 7 & -13.02 & 0.902 & 8.5 & \\
\hline 90 & 175317.77 & -280433.20 & 5 & $-13.93:$ & 0.777 & 0.97 & \\
\hline 91 & 175320.48 & -294032.30 & 17 & 0.00 & & & \\
\hline 92 & 175319.81 & -282714.67 & 5 & -13.93 & 0.523 & - & \\
\hline 93 & 175324.14 & -294948.45 & 14 & & 0.864 & 6.3 & \\
\hline 94 & 175333.15 & -283556.34 & - & 0.00 & & & \\
\hline 95 & 175335.38 & -282851.02 & 9 & -12.30 & 0.570 & 5.5 & \\
\hline 96 & 175357.16 & -292014.97 & 25 & $-12.45:$ & & & \\
\hline 97 & 175413.36 & -280516.82 & 7 & -13.25 & 0.677 & 11.5 & \\
\hline 98 & 175546.39 & -275338.91 & - & & 0.726 & 21.8 & IRAS 17480-2855 \\
\hline
\end{tabular}

${ }^{a}$ Diameters are taken from the $\mathrm{H} \alpha$ images when possible. Due to poor resolution, the [SIII] diameters are uncertain; those less than $5^{\prime \prime}$ are indicated by "-".

${ }^{b}$ Entries of 0.00 indicate that the PN was not recovered in the $\mathrm{H} \alpha$ imaging. Blank entries indicate that the PN was not within the $\mathrm{H} \alpha$ imaging survey area. Entries with ":" have large uncertainties due to contamination from the residuals of stars, or due to the faintness of the nebula.

${ }^{c}$ Entries of "-" indicate that the $\mathrm{H} \alpha$ line could not be measured in our spectra. Blank entries indicate that no spectrum was obtained and therefore the PN has not been confirmed spectroscopically. Entries with ":" are uncertain due to weak [NII] lines in the spectrum. Spectra were obtained either at the ESO/La Silla 1.5-m or CTIO 4-m telescopes.

${ }^{d}$ Radio fluxes were obtained at the Australia Telescope Compact Array and are reported in Van de Steene \& Jacoby (2001).

${ }^{e} \mathrm{~K}$-series entries are candidates initially suspected by Kohoutek (1994). Close coincidences $\left(<10^{\prime \prime}\right)$ with IRAS sources are indicated; 2 IRAS coincidences with somewhat larger separations (10-15") are shown with "?". 
$\mathrm{H} \alpha+[\mathrm{NII}]$ fluxes when available (see next section), an estimate of the fraction of flux that is purely $\mathrm{H} \alpha$ as derived from the PN spectrum, and their radio fluxes when available (Van de Steene \& Jacoby 2001). Finding charts are presented in the Appendix in Figs. A.1-A.9; the $\mathrm{H} \alpha$ images are presented when available since their resolution is far superior to the [SIII] images. Diameters in Table 2 are also preferentially taken from the $\mathrm{H} \alpha$ images. We refer to this set of objects with the designation of "JaSt" for consistency with previous papers.

\subsection{The Ho "re-survey"}

A primary goal of this project is to derive the chemical composition for $\mathrm{PNe}$ in the Galactic bulge from their emission-line ratios. Because of the generally heavy extinction to these objects, there are very few emission lines blueward of $\mathrm{H} \alpha ; \mathrm{H} \beta$ is extremely weak if it is visible at all. Therefore, we felt that an alternative method of deriving the extinction was necessary in order to de-redden the emission-line ratios. The approach we will adopt is to derive the extinction from the ratio of the radio continuum emission to the Balmer line emission, using $\mathrm{H} \alpha$ as the principal Balmer line. In the next paper of this series, we also demonstrate that one can derive a reliable reddening from the ratio of the Paschen P10 line to $\mathrm{H} \alpha$.

In addition, the photoionization modeling that is used to derive the chemical compositions is improved greatly if one has an estimate of the nebular flux and diameter, as these constrain the central star luminosity and nebula ionization structure. The central star luminosity is required to estimate its mass, and consequently, the age of its progenitor. With ages derived indirectly from the spectrum and photometric properties of the nebulae, combined with the chemical composition derived directly from the spectrum, we can deduce the chemical enrichment history for the stars in the Galactic bulge.

Consequently, we obtained direct images of the survey fields in $\mathrm{H} \alpha$ in order to measure the fluxes and diameters of the nebulae using the NOAO CCD Mosaic camera (Muller et al. 1998) on the KPNO (now, WIYN Observatory) 0.9-m telescope. This facility offers a unique combination of wide field $\left(59^{\prime} \times 59^{\prime}\right)$, excellent sensitivity, and good sampling $\left(0.43^{\prime \prime}\right.$ pixels). We observed the Galactic center fields listed in Table 1 on the nights of 10-13 June 1999. We used an $\mathrm{H} \alpha+$ [NII] filter having a central wavelength of $6569 \AA$ and a $F W H M$ of $80 \AA$ and an $R$-band filter to serve as an "off-band" filter. To derive the photometric calibration, each night we observed 4-5 of the following spectrophotometric standard stars: HZ-44, Wolf 1346, BD+28 4211, Feige 66, Feige 110, PG 1545+035. Flux measurements were made on the difference images $(\mathrm{H} \alpha$ minus $R$-band) in order to minimize the degree of stellar contamination.

Because there are gaps between the CCDs, we observed each of the 16 fields in a dithered pattern of 5 images per field. Each of the 5 exposures was $360 \mathrm{~s}$, for a total on-band exposure of $30 \mathrm{~min}$. Each of the $R$-band exposures was $36 \mathrm{~s}$, for a total "off-band" exposure of $3 \mathrm{~min}$. The data were reduced using the IRAF suite of tasks in the MSCRED package (Valdes 1998), which, after the combination of each dithered
Table 3. Optical positions and $\mathrm{H} \alpha$ fluxes for PNe discovered in $\mathrm{H} \alpha$ survey.

\begin{tabular}{lllcl}
\hline \hline JaSt 2 & $\begin{array}{l}\text { RA } \\
\text { (J2000.0) }\end{array}$ & $\begin{array}{l}\text { Dec } \\
\text { (J2000.0) }\end{array}$ & $\begin{array}{c}\text { Diam } \\
(\operatorname{arcsec})\end{array}$ & $\begin{array}{l}\text { Log Flux } \\
\text { H } \alpha+[\mathrm{NII}]\end{array}$ \\
\hline $1^{b}$ & 1734.29 .3 & -290203.0 & 50 & -12.94 \\
2 & 173844.8 & -280644.4 & 12 & -13.78 \\
3 & 174109.6 & -282800.0 & 6 & -13.70 \\
4 & 174227.9 & -271331.4 & 30 & -13.54 \\
5 & 174348.9 & -265330.0 & 18 & -13.58 \\
$6^{c}$ & 175001.5 & -293315.8 & 15 & -12.82 \\
7 & 175144.0 & -301249.2 & 39 & -12.28 \\
8 & 175203.5 & -291634.5 & 32 & -12.66 \\
9 & 175247.6 & -291721.9 & 9 & -13.42 \\
10 & 175317.2 & -283604.0 & 10 & -12.56 \\
11 & 175327.0 & -290817.5 & 9 & -13.06 \\
12 & 175327.5 & -281147.8 & 22 & -13.14 \\
13 & 175335.6 & -285225.7 & 19 & -13.26 \\
14 & 175340.7 & -294236.1 & 50 & -12.18 \\
15 & 175423.5 & -283450.7 & 19 & -12.74 \\
16 & 175523.0 & -282012.9 & 7 & -13.48 \\
17 & 175536.5 & -282531.0 & 17 & -12.70 \\
18 & 175554.9 & -280556.5 & 18 & -13.18 \\
19 & 175633.8 & -283035.6 & 5 & -13.61 \\
20 & 175635.9 & -285725.6 & 20 & -12.82 \\
\hline
\end{tabular}

${ }^{a}$ Entries all have very large uncertainties because of the degree of crowding from stars over the large diameters of these nebulae.

${ }^{b}$ Large, possibly bi-polar.

${ }^{c}$ Unusually bright knot just off-center; possibly coincident with IRAS 17468-2932 and 1RXS J175001.8-293316.

set, yields a single image of approximately $61^{\prime} \times 61^{\prime}$. Thus, these fields are about $28 \%$ smaller in area on the sky than the Schmidt [SIII] fields, and some PNe were not recovered at $\mathrm{H} \alpha$. Those PNe that were recovered at $\mathrm{H} \alpha$ will have a flux entry in Table 2, while those that were too faint to be recovered have a flux listed as exactly 0.00 . Those PNe that fell outside the $\mathrm{H} \alpha$ fields are flagged by a blank entry as the flux. Images of those PNe visible at $\mathrm{H} \alpha$ are shown in Figs. A.1-A.9; if not visible in $\mathrm{H} \alpha$, the chart illustrates the [SIII] image.

\subsection{New PN candidates in $\mathrm{H} \alpha$}

At modest levels of extinction, $\mathrm{H} \alpha$ provides good detection sensitivity for finding faint PNe. To guard against emission-line stars, only objects that are non-stellar should be considered viable PN candidates. With these criteria, 20 additional PN candidates were identified in the $\mathrm{H} \alpha$ survey. Until confirmation spectra of these are obtained, we consider these objects to be candidate PNe. On the other hand, the morphologies of at least half of the $\mathrm{H} \alpha$ candidates look very much like true PNe.

Table 3 lists the new $\mathrm{H} \alpha$ candidates, their coordinates, diameters, and $\mathrm{H} \alpha+[\mathrm{NII}]$ fluxes. We refer to this new set of objects with the designation "JaSt 2" to distinguish them from the [SIII] sample. Figures A.10-A.13 provide finding charts. 


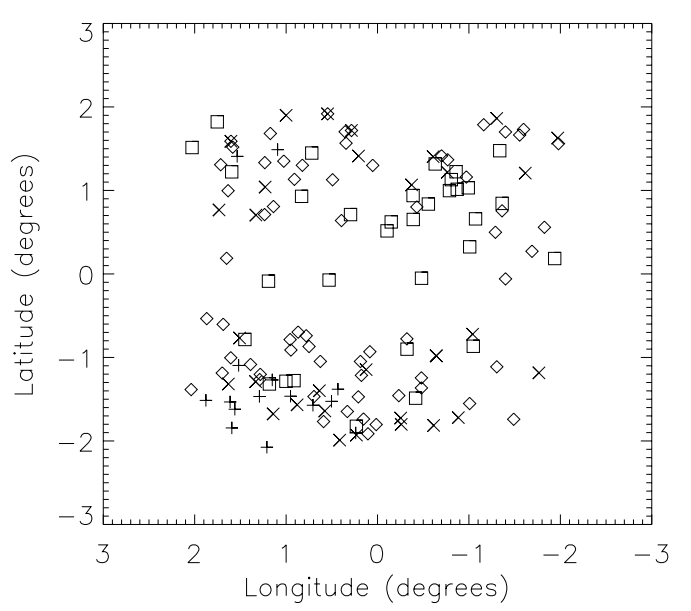

Fig. 1. The location of the new PNe discovered in the [SIII] survey (squares), the new PNe discovered in the $\mathrm{H} \alpha$ "re-survey" (plus signs), those $\mathrm{PNe}$ that were confirmed spectroscopically (diamonds) and those PNe that were previously known in the survey area (crosses). Note the lack of objects within a half-degree of the Galactic plane, where the extinction becomes extreme.

As Fig. 1 illustrates, the $\mathrm{H} \alpha$ candidates are found predominantly at the most negative latitudes and in selected regions, presumably where the extinction is least severe.

\section{Discussion}

\subsection{Verification spectra}

In order to assess the validity of the identification of the candidates as true PNe, we obtained far red (5000-10000 ̊̊) spectra of as many objects as possible during several runs at the ESO 1.5-m during the June 1995 and July 1996 observing seasons, and a single run at the CTIO 4-m in June of 1997. Note that observations at these wavelengths present some extra challenges due to very severe sky brightness and CCD fringing. It is difficult, therefore to obtain spectra with accurate sky subtraction. The details of these observations are given in the next paper.

In all, we observed 73 candidates spectroscopically. Of these, 63 proved to be genuine emission-line sources, exhibiting emission at [SIII] $\lambda 9532$ as a minimum requirement. The remaining 10 objects that we observed showed nothing at all, possibly because they were too faint, the coordinates were inaccurate, the telescope pointing was off, or these are not PNe. In addition, we did not have enough telescope time to observe 24 candidates. Based on the very high success rate thus far, though, most of these are likely to be true PNe as well. The notes column in Table 2 indicate whether the object was verified spectroscopically.

Of the 34 candidates that were not confirmed via spectra, we must be most skeptical of the 17 candidates that were not recovered either in the radio or the $\mathrm{H} \alpha$ re-surveys (having a 0.00 in Col. 5 and blank in Col. 7). Most of these candidates are also among the faintest, and so, the lack of secondary detections may be due to the flux limits of the radio and $\mathrm{H} \alpha$ surveys. The exceptions are candidates 53 and 91, for which the [SIII] images appear moderately bright.

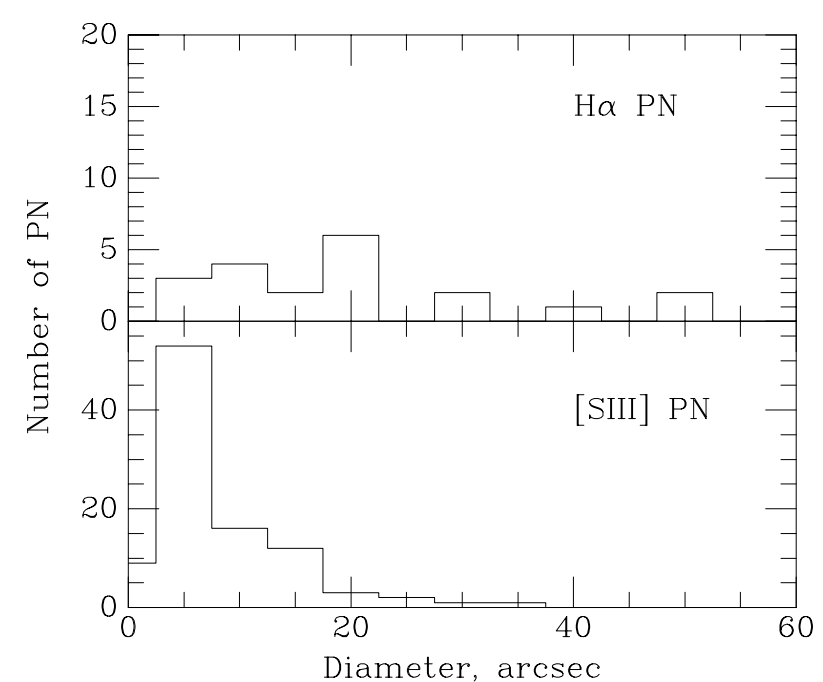

Fig. 2. Histograms of the diameter distribution of the new PNe found in the [SIII] and $\mathrm{H} \alpha$ surveys. Some of the objects are as large as 2 pc. Thus, in some cases, we are finding very old PNe. On average, the $\mathrm{H} \alpha$ survey is more sensitive to larger PNe.

On the other hand, we have very high confidence in those 5 candidates of the 34 without spectra that were recovered in the $\mathrm{H} \alpha$ re-survey (JaSt 12, 72, 84, 87, 96). These objects all must be emission-line sources at both [SIII] and $\mathrm{H} \alpha+[\mathrm{NII}]$, plus they have sizes and morphologies consistent with Galactic center PNe.

For a subset of $\sim 12$ objects having relatively high quality spectra, we see many emission-lines that offer insight into the chemical composition of the nebulae. We will report on these in a subsequent paper.

\subsection{Spatial distribution of the new $P N e$}

Figure 1 illustrates the location of the new PNe with respect to their positions in the Galaxy. The PNe discovered in this survey extend the identifications of the previously known $\mathrm{PNe}$ much closer to Galactic plane. The central \pm 0.5 degree remains a challenge where the extinction becomes very large. A few of our new identifications, though, are right along the plane, suggesting the presence of holes in the dust distribution. Alternatively, these objects may be foreground to the Galactic center.

\subsection{Diameters of the new $\mathrm{PNe}$}

Diameters are only approximate, even for the best cases. For objects smaller than $5^{\prime \prime}$ in [SIII] and $2^{\prime \prime}$ in $\mathrm{H} \alpha$, the spatial resolution of the images is not adequate for a measurement; we do not present diameters for these small objects. Larger objects also have uncertain diameters because the outer isophotes of PNe can be too faint to see. In addition, PNe are far more complex than a simple sphere, and so, a single parameter cannot characterize the size. Nevertheless, even rough estimates of the nebula sizes are valuable for later photoionization model analysis and for understanding the selection effects in the identifications. 


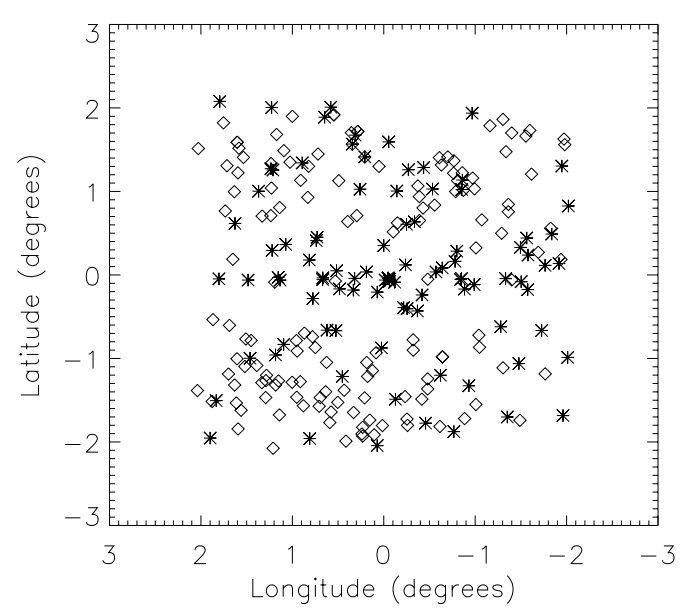

Fig. 3. The location of known OH/IR stars (asterisks) compared to the new $\mathrm{PNe}$ discovered in the [SIII] and $\mathrm{H} \alpha$ surveys (diamonds).

Figure 2 shows the distribution of diameters for the PNe identified in [SIII] and $\mathrm{H} \alpha$. Assuming that all these PNe are truly at the distance of the Galactic center $(7.9 \mathrm{kpc}$; Eisenhauer et al. 2003), a diameter of $10^{\prime \prime}$ corresponds to $0.38 \mathrm{pc}$. While most of the PNe are fairly small, a few are quite large, especially those found in $\mathrm{H} \alpha$. In fact, the largest PNe are nearly $2 \mathrm{pc}$ across, demonstrating that our survey is sufficiently sensitive to find very old, low surface brightness PNe.

The PNe found only in $\mathrm{H} \alpha$ tend to be larger, on average, than those identified first in [SIII]. This bias suggests that the [SIII] survey is not nearly as sensitive to the low surface brightness emission. Because the sky brightness at $9530 \AA$ is much greater than at $6560 \AA$, and because the 0.9 -m telescope with the Mosaic Camera is about 10 times more sensitive than the smaller Schmidt and its thick CCD, the greater depth of the $\mathrm{H} \alpha$ survey is not surprising.

\subsection{OH/IR stars}

Figure 3 shows the Galactic distribution of OH/IR stars from Sevenster et al. (1997) superposed on our PN distribution. These $1612 \mathrm{Mhz} \mathrm{OH}$ maser data extend right into the plane because extinction from dust is not a factor. Outside the central \pm 0.5 degree, the distribution of $\mathrm{OH} / \mathrm{IR}$ stars is comparable to that of the PNe. The very high detection rate of OH/IR stars within $\sim 0.25$ degrees of the plane $( \pm 30 \mathrm{pc})$, though, exceeds any reasonable expectation that all of these can be progenitors of PNe. Instead, some of these must be derived from a very different population. Consequently, we caution against the general use of $\mathrm{OH} / \mathrm{IR}$ stars as tracers of post-AGB stars.

\subsection{The number of $P N e$ In the Galactic bulge}

Based on the density of IRAS and OH/IR sources having IR colors similar to $\mathrm{PNe}$, we anticipated that $\sim 320 \mathrm{PNe}$ are present in our fields within the Galactic bulge. The expected number of PNe in our survey region can also be estimated from the luminosity of the Galactic bulge and the production rate of PNe as seen in other galaxies. Dwek et al. (1995) estimated the bolometric luminosity of the central $10 \times 10$ degrees of the bulge at $5.3 \times 10^{9} L_{\odot}$. We used their model G0, corrected for its underestimation relative to the data within 3 degrees of the center, to determine that the luminosity fraction appropriate to our $4 \times 4$ degree survey is $19.2 \%$. This corresponds to $1.02 \times 10^{9} L_{\odot}$. However, Dwek et al. adopted a distance to the Galactic center of $8.5 \mathrm{kpc}$, whereas more recent estimates (e.g., Eisenhauer et al. 2003) find this value to be $7.9 \mathrm{kpc}$. Correcting for this distance differential, we adopt a bolometric luminosity for our survey region of $0.89 \times 10^{9} L_{\odot}$.

For nearby galaxies, Ciardullo (1995) finds that the production rate for bright $\mathrm{PNe}$ (within 2.5 mags of the most luminous PNe in the galaxy) varies between $\sim 10 \times 10^{-9}$ and $\sim 50 \times 10^{-9}$ PNe per unit of bolometric solar luminosities. The latter number represents the case where all stars become $\mathrm{PNe}$ and is approached for young populations (e.g., LMC and SMC). For the older populations typically seen near the centers of galaxies, this rate is $\sim 20 \times 10^{-9}$. Thus, the Galactic bulge is expected to have $\sim 18$ bright PNe. The luminosity function for PNe can be used to extrapolate this number to include the faint $\mathrm{PNe}$, but with significant uncertainty. That correction factor is 10.1 , suggesting a total of $\sim 180 \mathrm{PNe}$ in the bulge survey zone. However, this number could be as high as 450 if the Galactic Center is dominated by a young population of stars.

Because the uncertainties in both estimation methods are difficult to establish, we simply average these two numbers. Thus, we expect there to be $250 \mathrm{PNe}$ in the surveyed region of the Galactic bulge.

Our near-IR search identified $140 \mathrm{PNe}$, for which $\sim 25 \%$ were already known. The follow-up $\mathrm{H} \alpha$ survey identified another 20 candidates, bringing the total count to $160 \mathrm{PNe}$, or $64 \%$ of the $\sim 250 \mathrm{PNe}$ expected. There must certainly be fainter, more extincted, PNe to be found, as evidenced by our poor detection rate within 0.5 degrees of the Galactic plane. Nevertheless, we now have identified nearly two-thirds of the predicted total number of PNe.

\section{Conclusions}

With this near-IR survey for PNe close to the Galactic center, we have shown that:

1. Highly extincted PNe can be found effectively by surveying in the emission line of [SIII] 29532, especially if augmented by a survey in $\mathrm{H} \alpha$.

2. The census of PNe within 2 degrees of the Galactic center is about two-thirds complete.

3. There are now many target PNe for follow-up chemical composition analysis but most of these are so highly reddened that their blue emission lines are likely to be unobservable. Thus, optical studies will be severely hampered except for a few of the brighter, less extincted, objects. We will present our spectroscopic results for several of these $\mathrm{PNe}$ in a subsequent paper. The remaining PNe to be found in the inner Galactic bulge will be even fainter and/or more seriously reddened.

Acknowledgements. We wish to thank Stuart Pottasch who initially suggested that we carry out a survey of this nature. 


\section{Appendix A: Finding charts}

$\mathbf{N}$

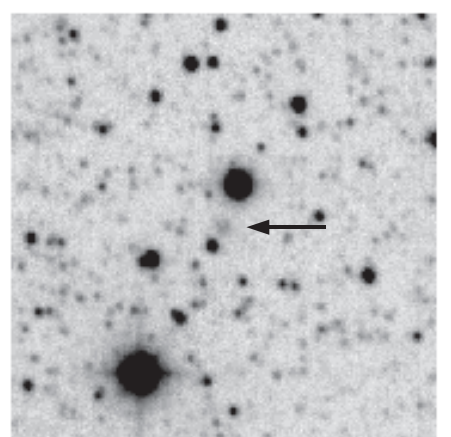

1

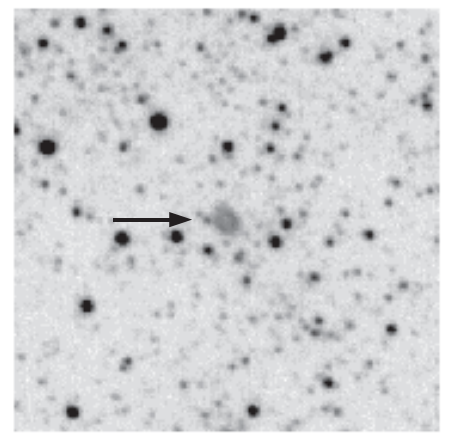

$\mathbf{E}$

4

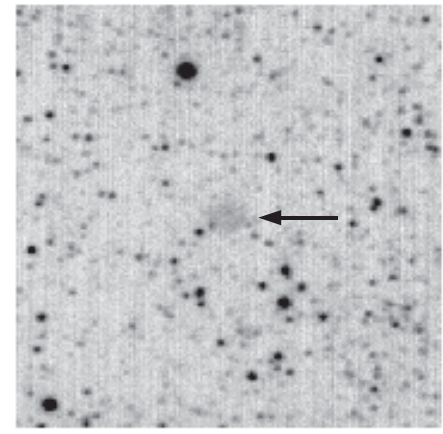

7

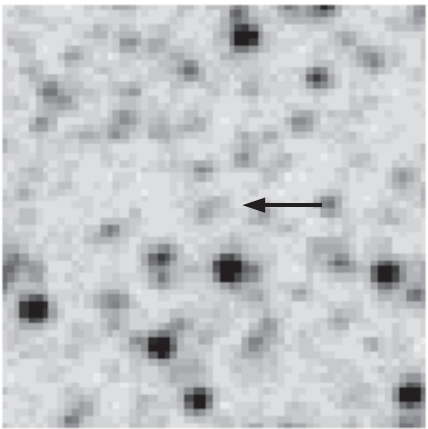

10

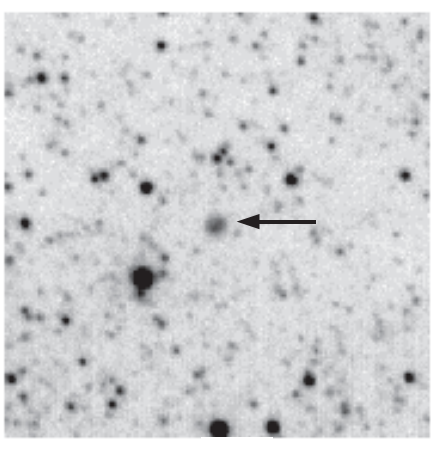

2

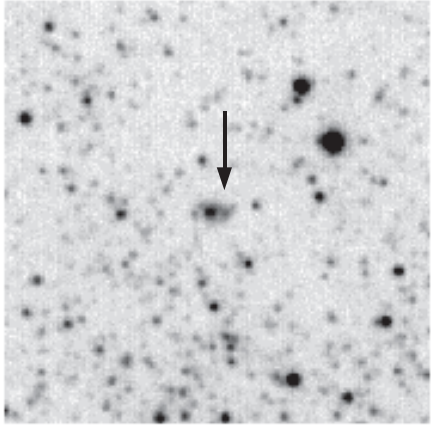

5

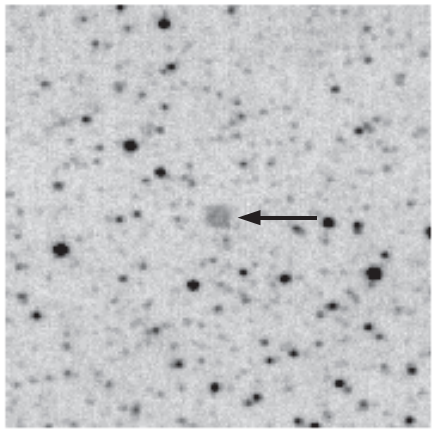

8

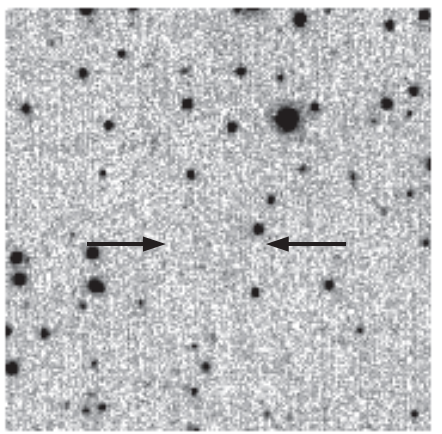

11

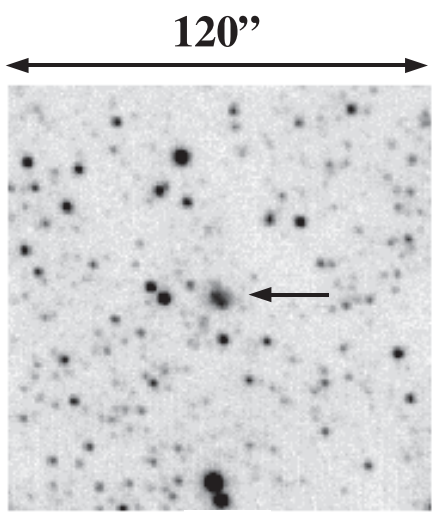

3

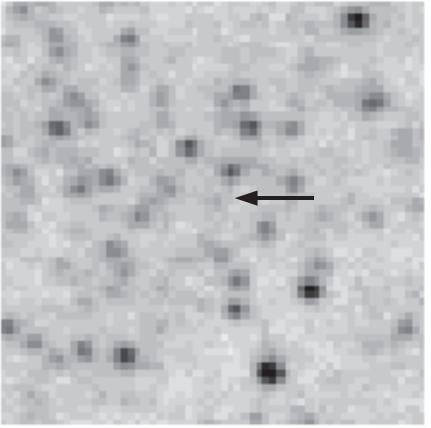

6

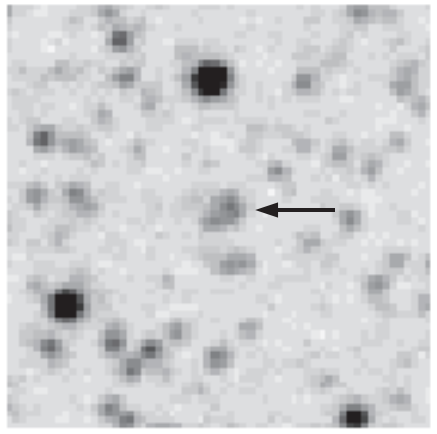

9

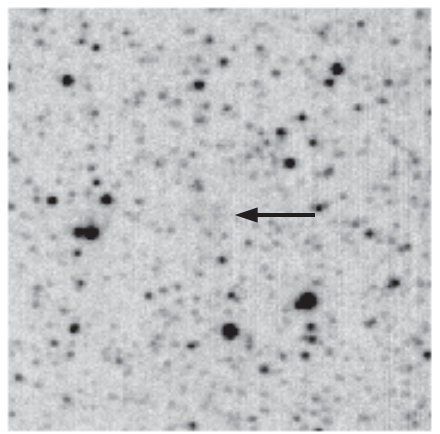

12

Fig. A.1. Finding charts for the new Galactic bulge PN identified as number 1-12. Each chart is 2 arcmin on a side. Each PN is denoted by an arrow ( 2 arrows for large or ambiguous cases) and is centered in the box, except when it was near the very edge of the survey image. The $\mathrm{H} \alpha$ images are shown whenever possible because of their superior spatial resolution. The [SIII] images are used for PN 6, 9, and 10. 


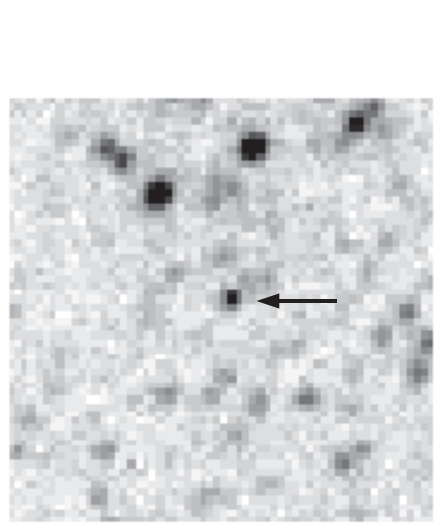

13

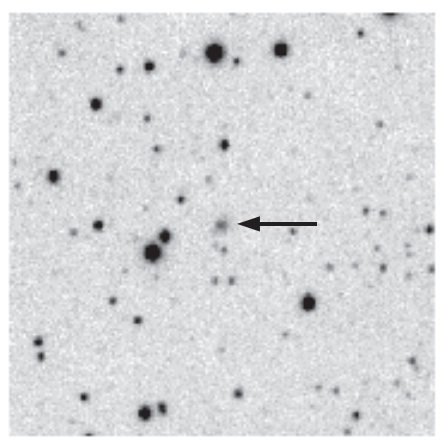

$\mathbf{E}$

16

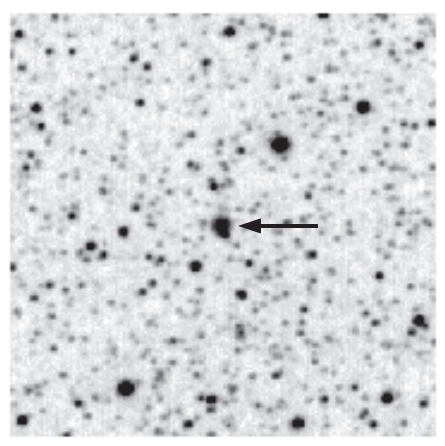

19

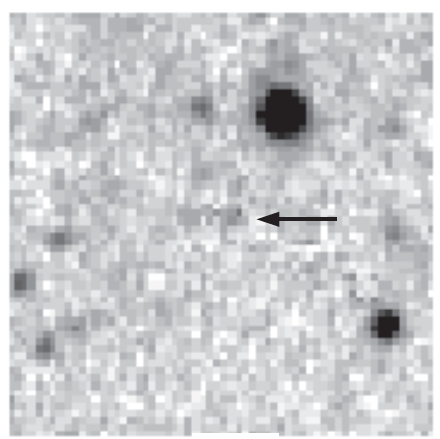

22

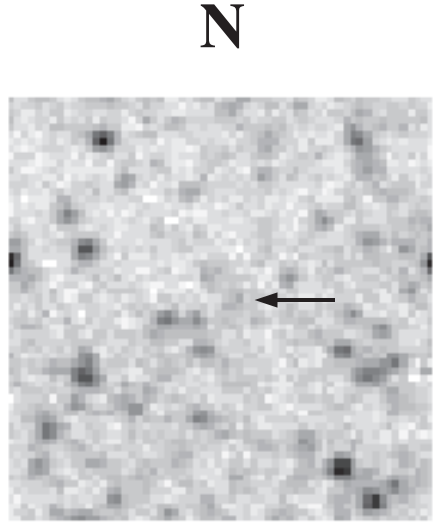

14

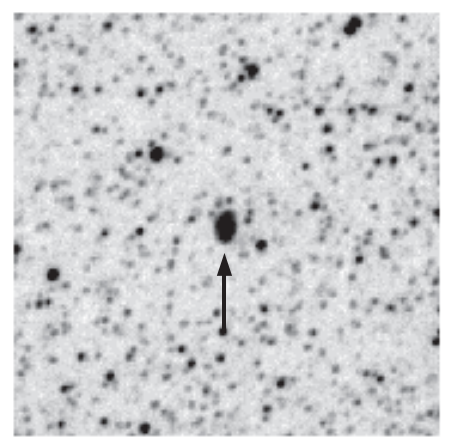

17

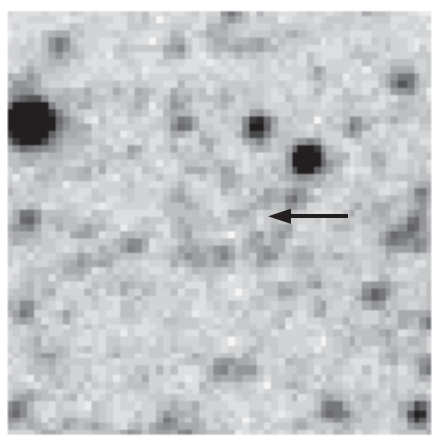

20

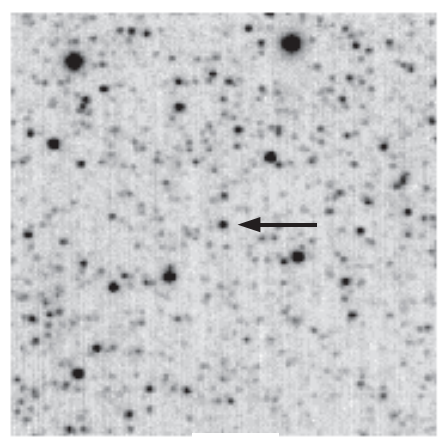

23

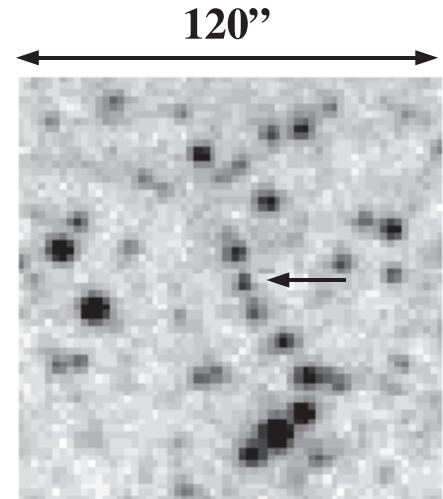

15

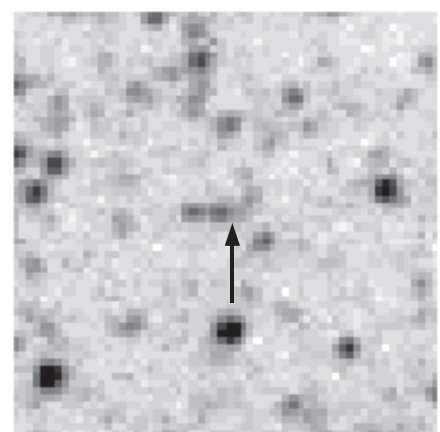

18

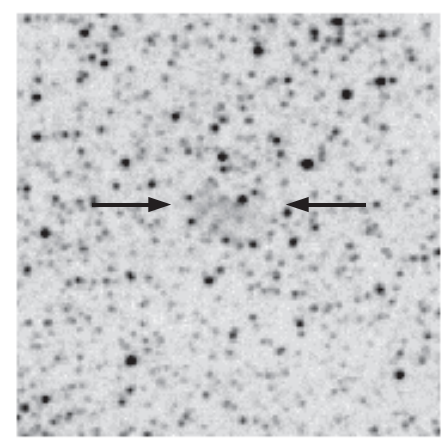

21

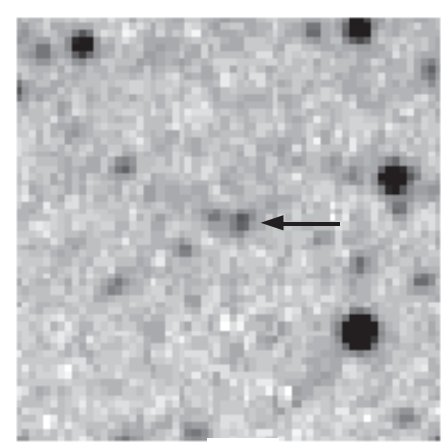

24

Fig. A.2. As for Fig. A.1, but showing the finding charts for PN 13-24. [SIII] images are shown for PN 13, 14, 15, $18,20,22$, and 24. 


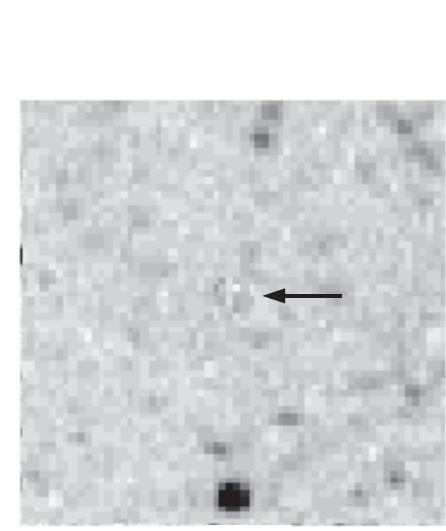

25

$\mathbf{E}$

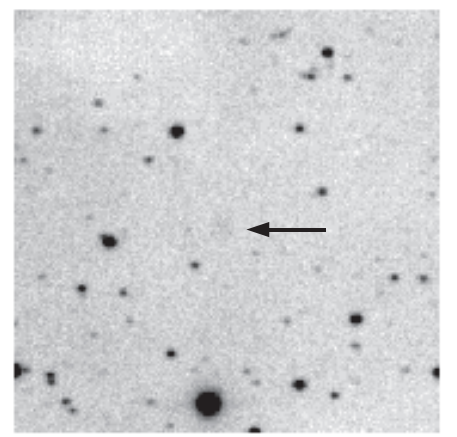

31

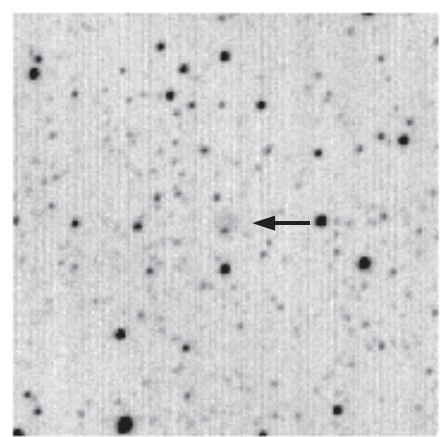

34

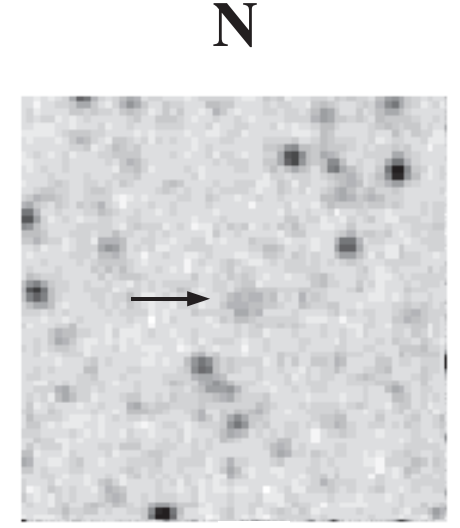

26

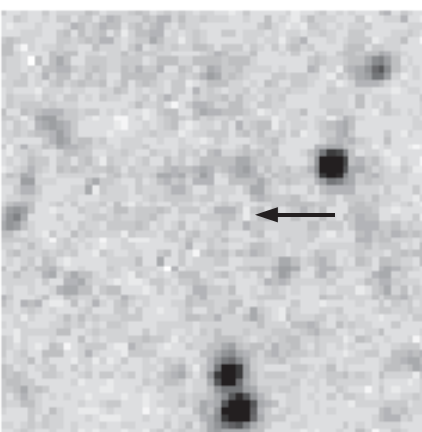

29

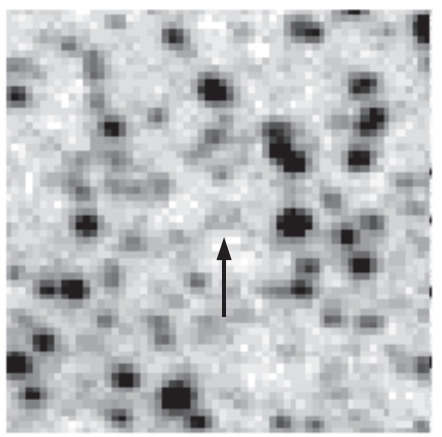

32

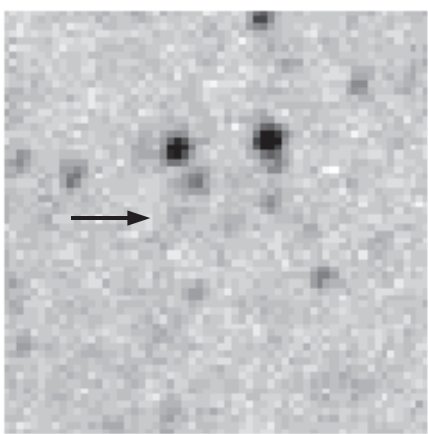

35

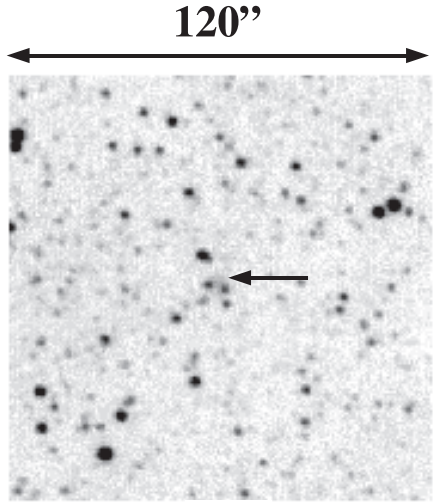

27

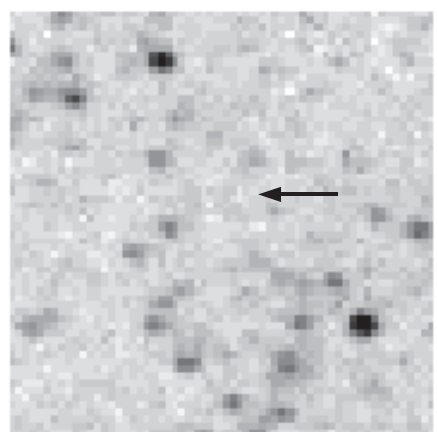

30

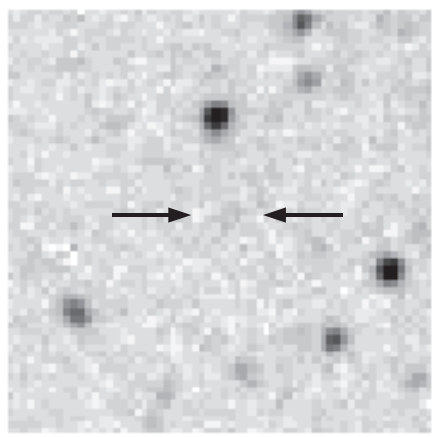

33

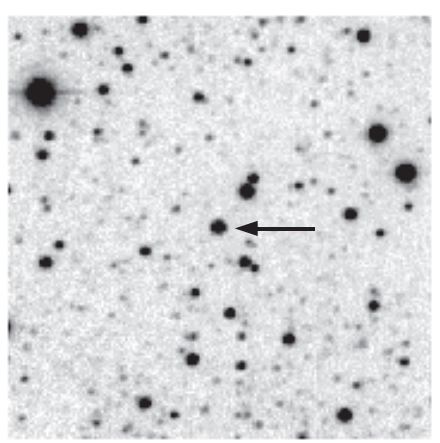

36

Fig. A.3. As for Fig. A.1, but showing the finding charts for PN 25-36. [SIII] images are shown for PN 25, 26, 29, 30, 32, 33, and 35. Note that PN 28 is not shown, as it was later removed from the list of confirmed PN. 


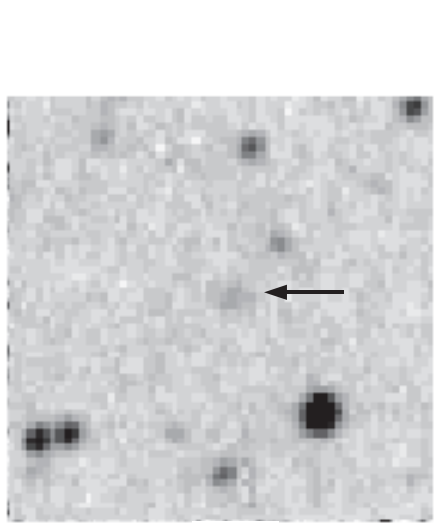

37

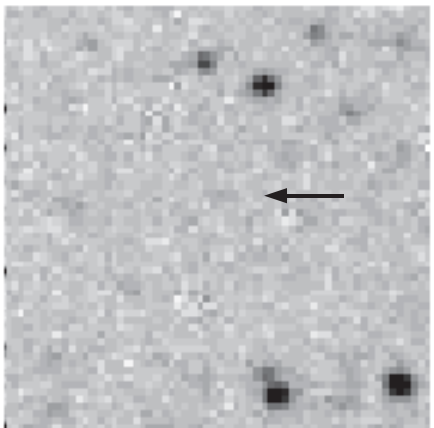

$\mathbf{E}$

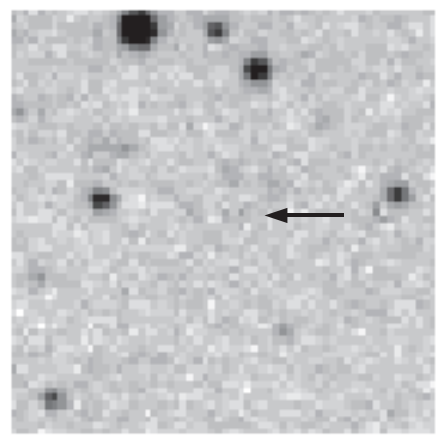

43

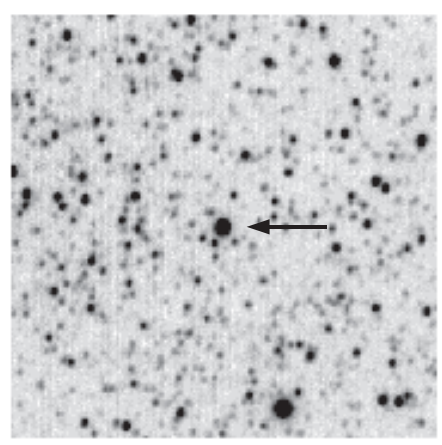

46

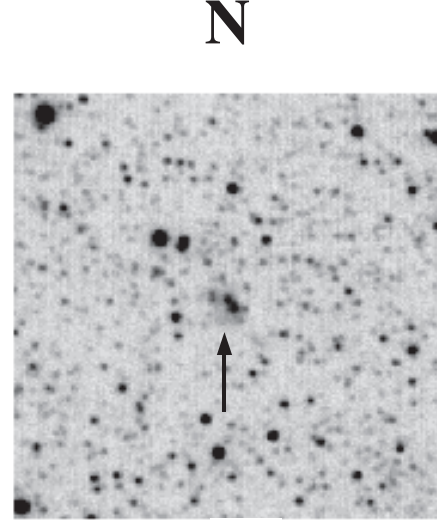

38

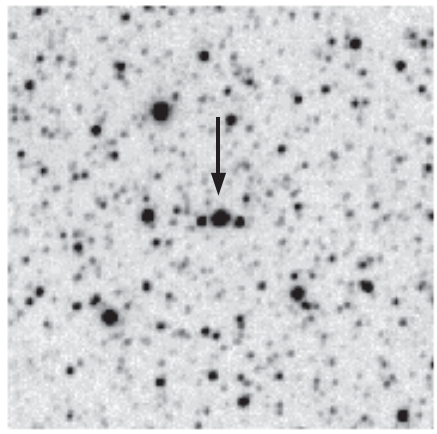

41

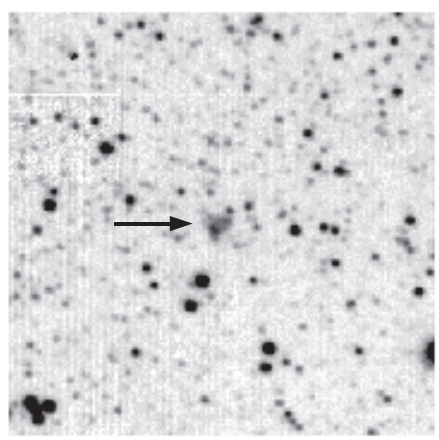

44

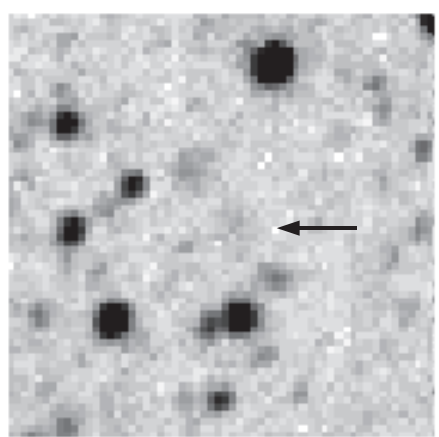

47

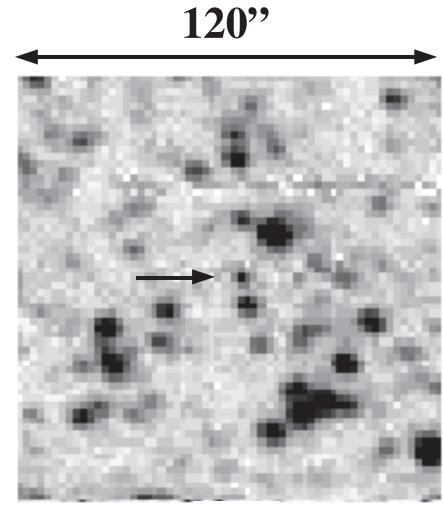

39

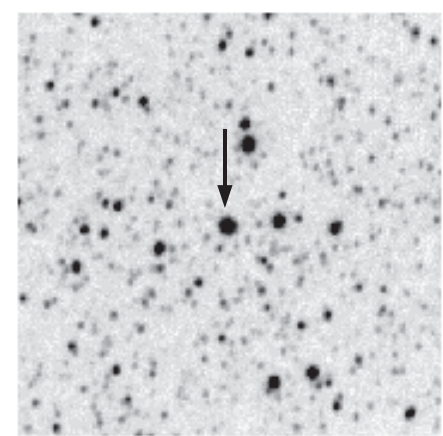

42

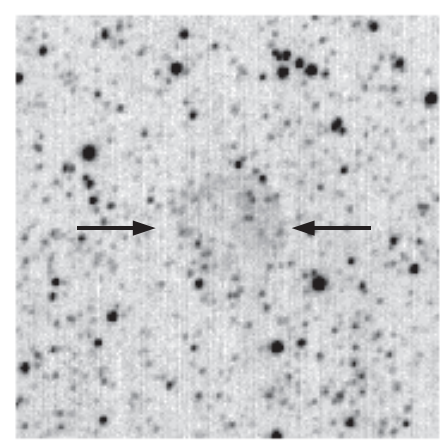

45

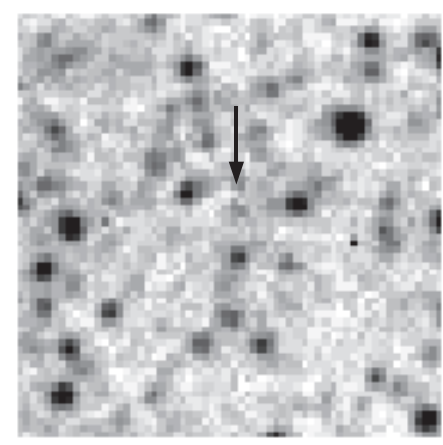

48

Fig. A.4. As for Fig. A.1, but showing the finding charts for PN 37-48. [SIII] images are shown for PN 37, 39, 40, 43, 47, and 48. 


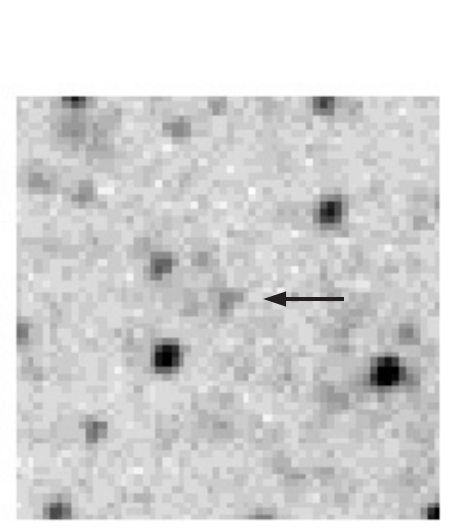

49

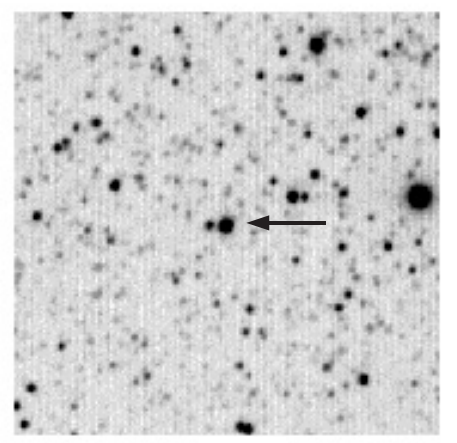

$\mathbf{E}$

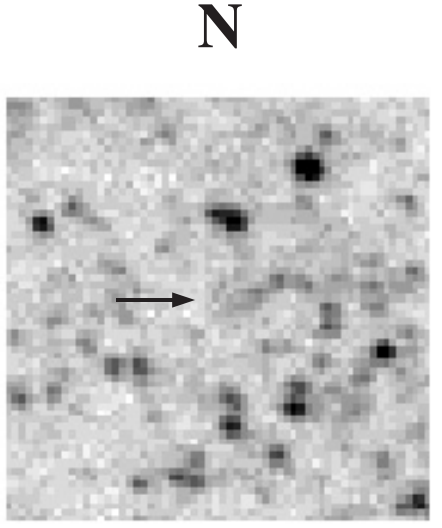

50

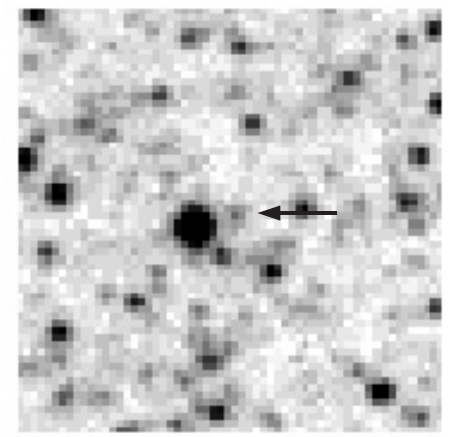

53

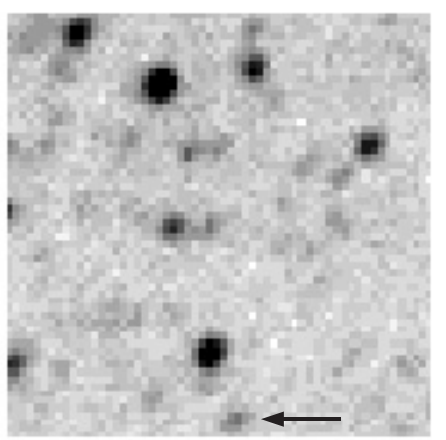

56

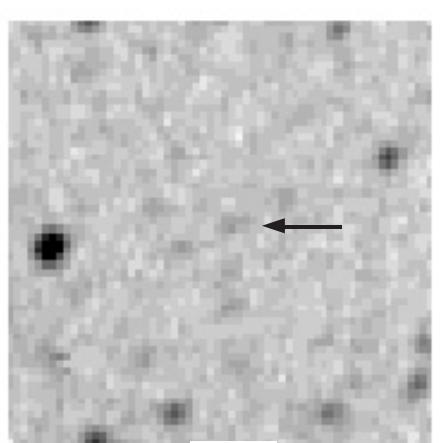

59

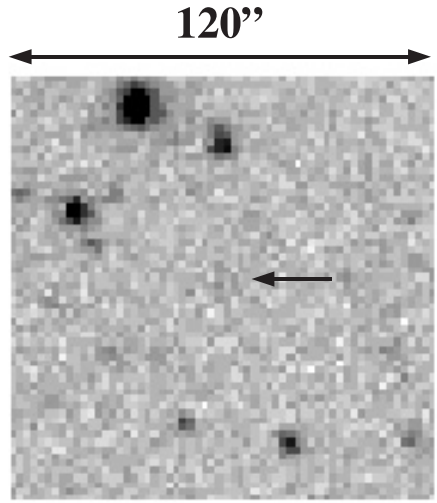

51

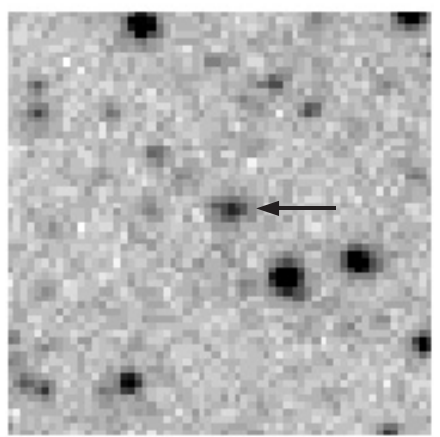

54

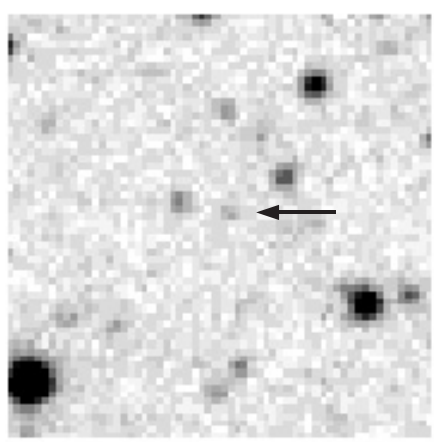

57

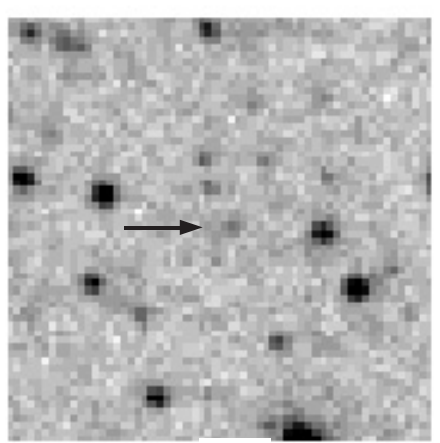

60

Fig. A.5. As for Fig. A.1, but showing the finding charts for PN 49-60. [SIII] images are shown for PN 49, 50, 51, 53, 54, 56, 57, 59, and 60. 


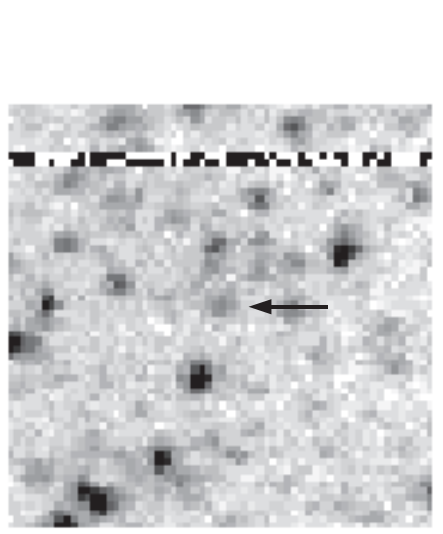

61

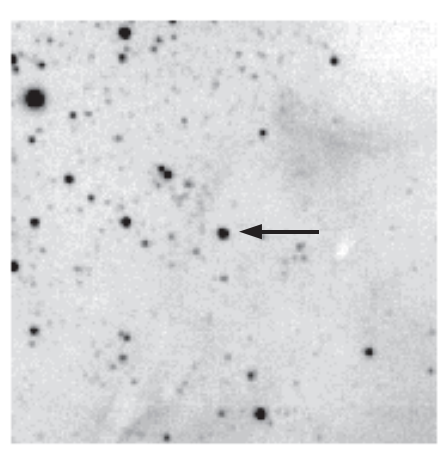

64

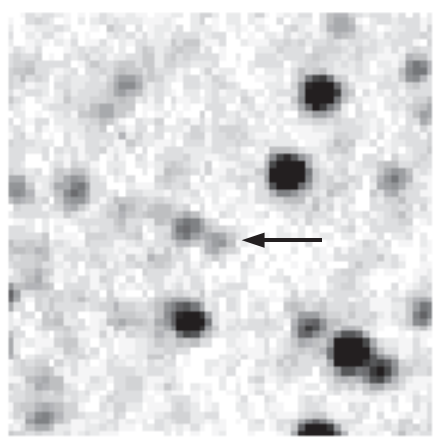

67

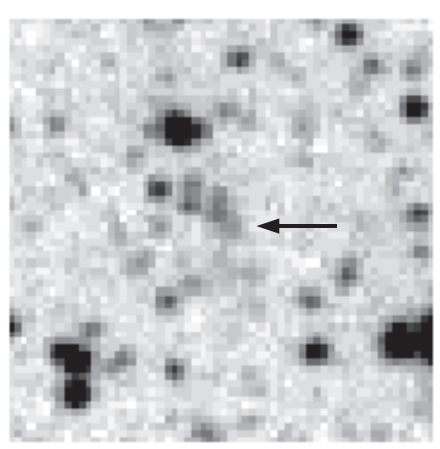

70

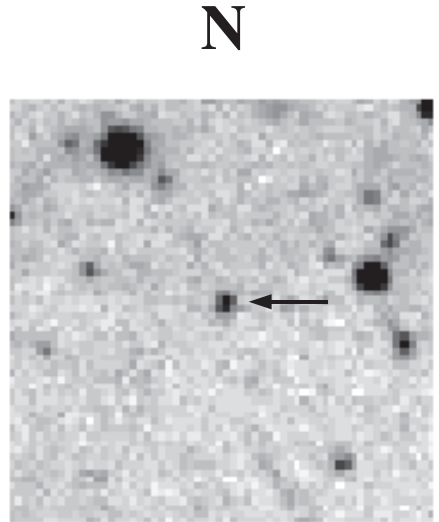

62

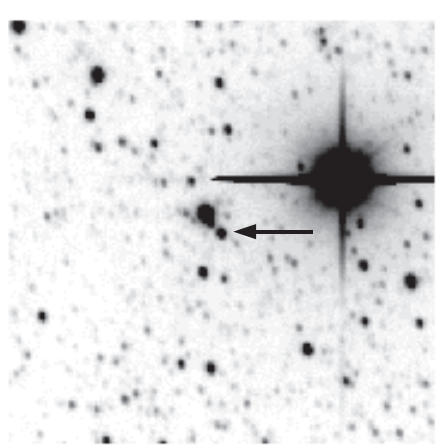

65

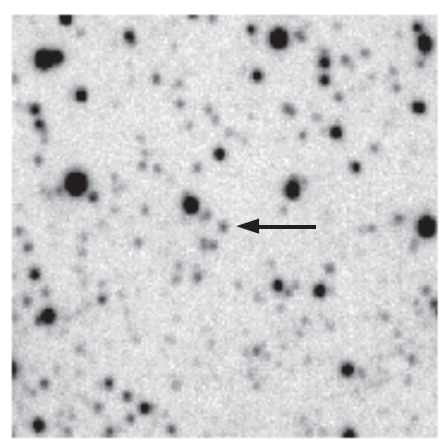

68

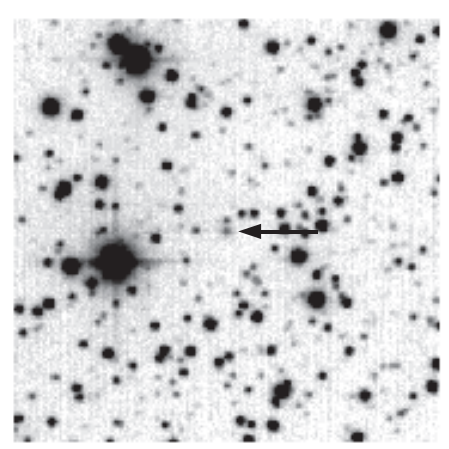

71

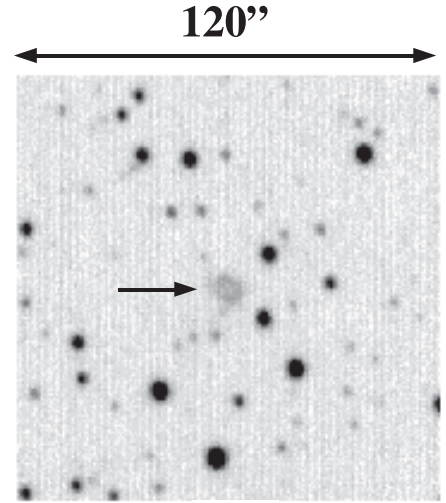

63

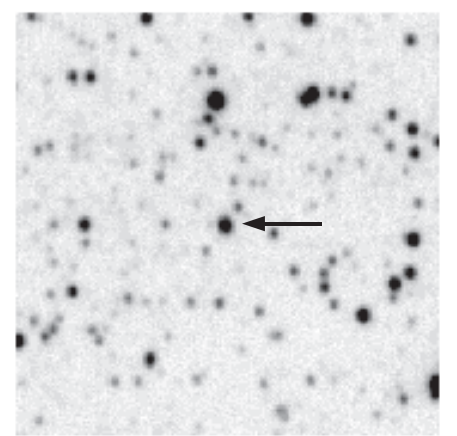

66

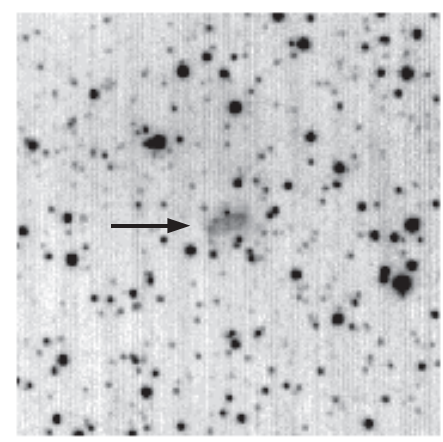

69

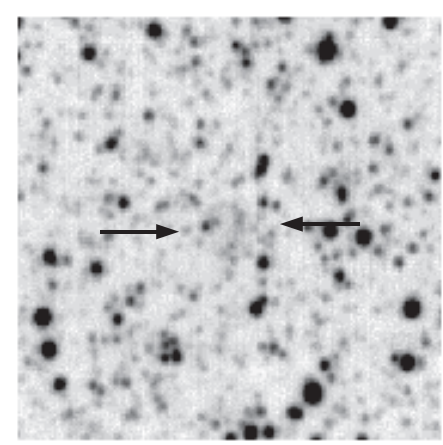

72

Fig. A.6. As for Fig. A.1, but showing the finding charts for PN 61-72. [SIII] images are shown for PN 61, 62, 67, and 70. 


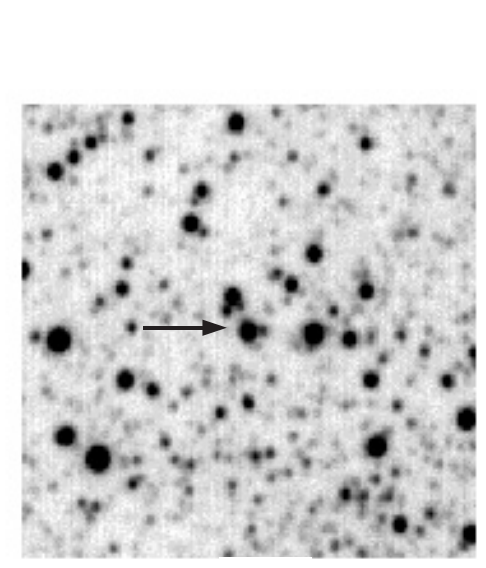

73

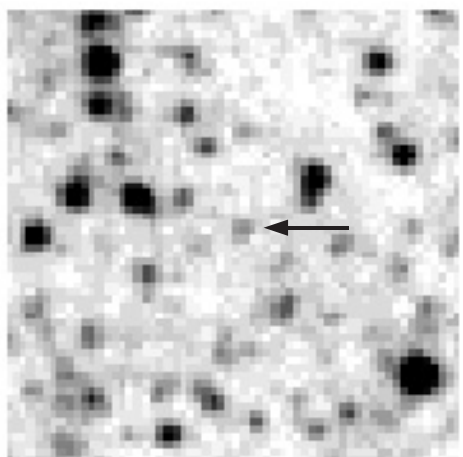

E

76

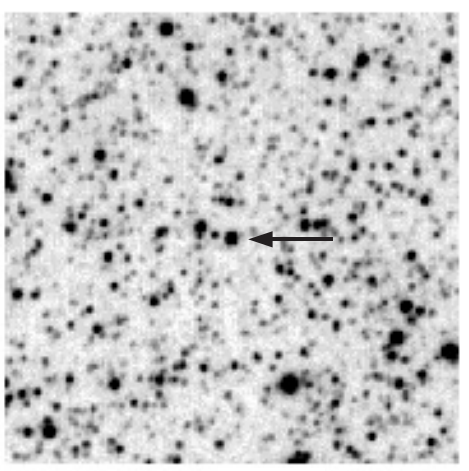

79

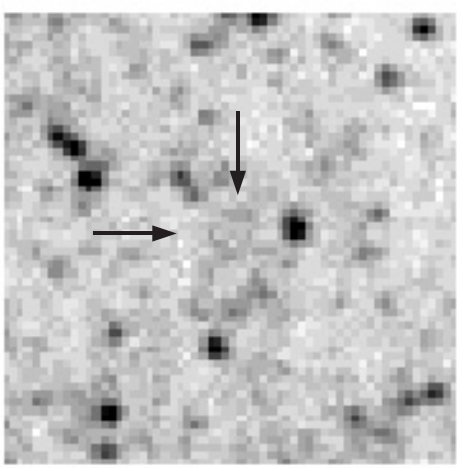

82

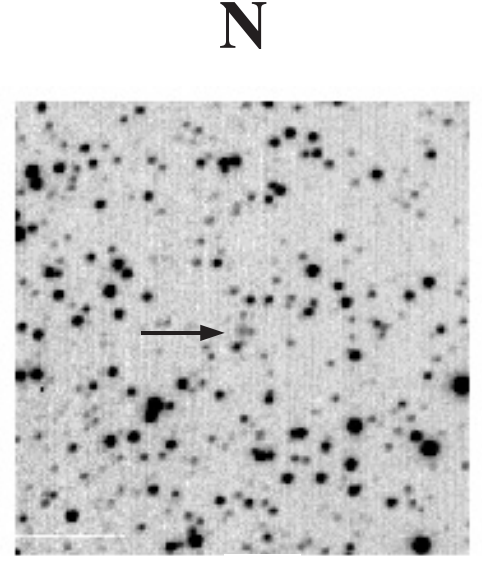

74

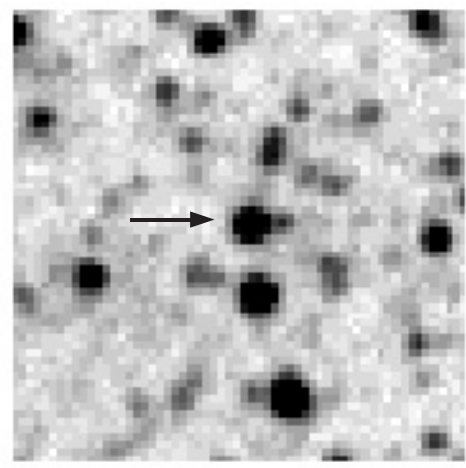

77

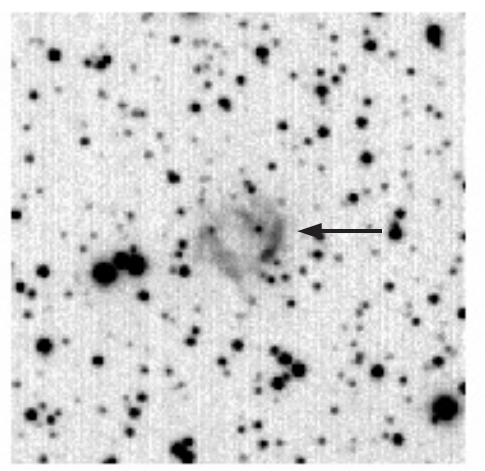

80

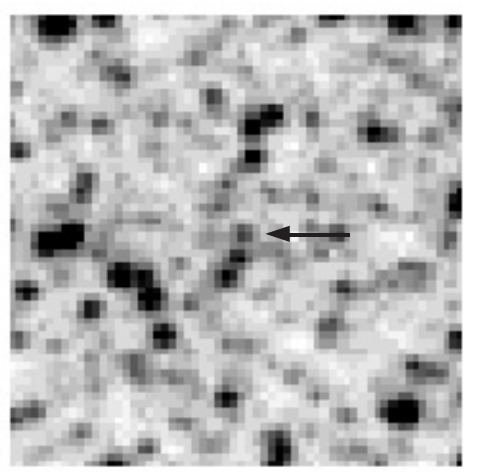

83

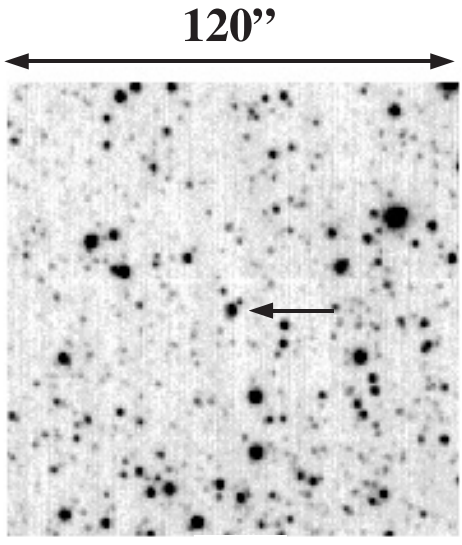

75

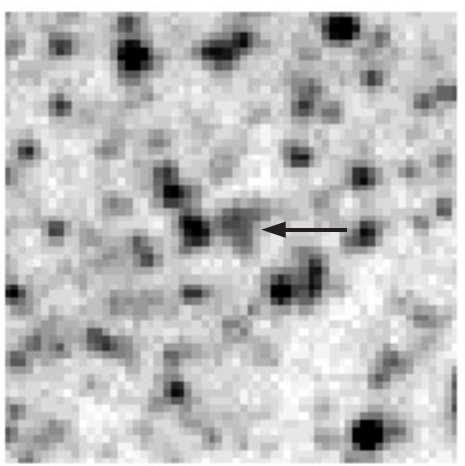

78

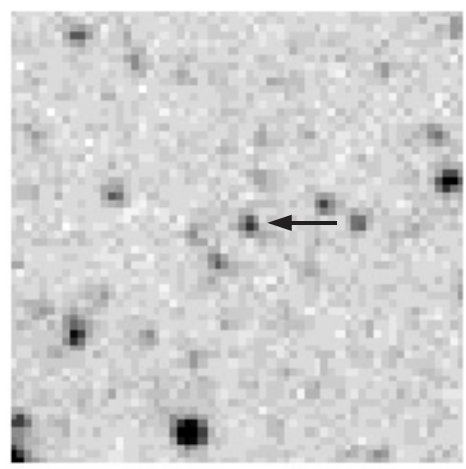

81

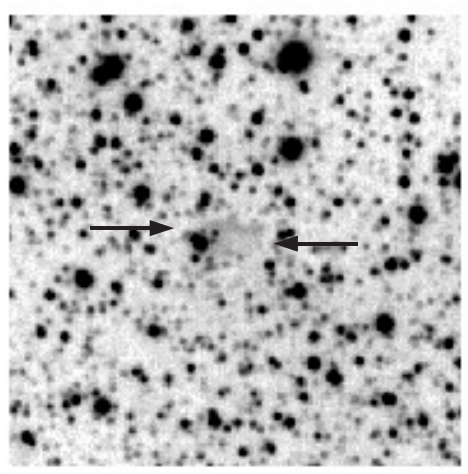

84

Fig. A.7. As for Fig. A.1, but showing the finding charts for PN 73-84. [SIII] images are shown for PN 76, 77, 78, 81, 82, and 83. 


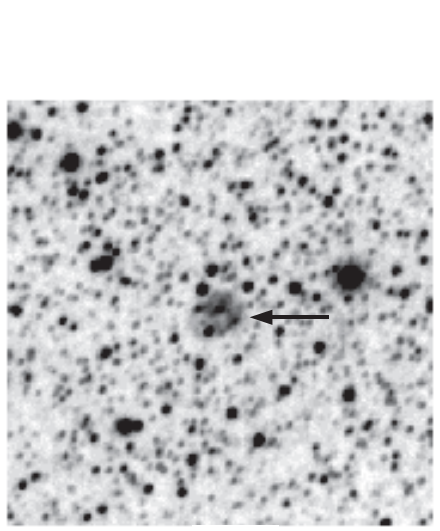

85

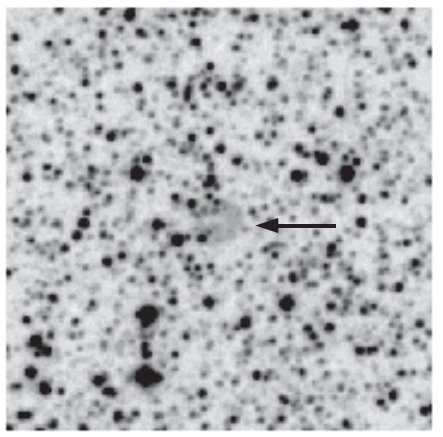

$\mathbf{E}$

88

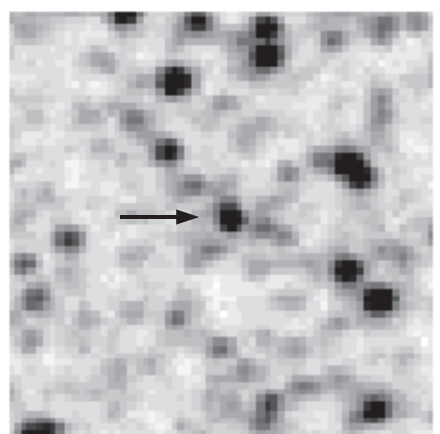

91

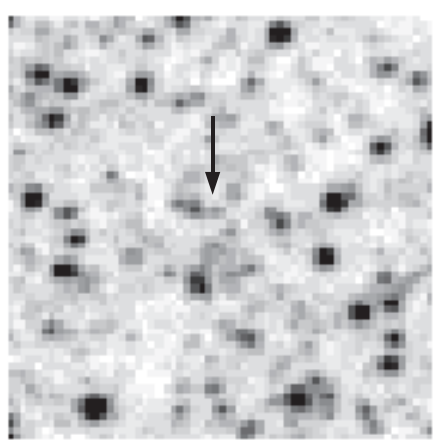

94

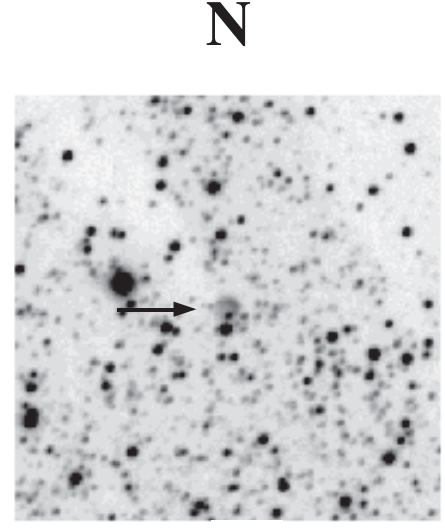

86

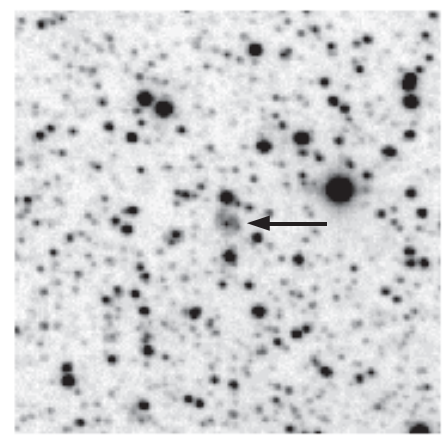

89

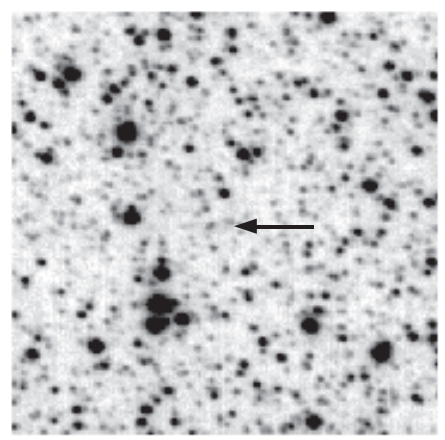

92

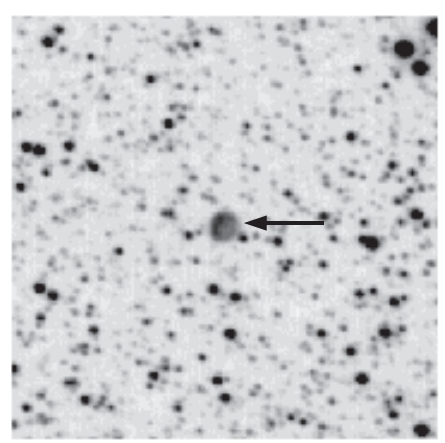

95

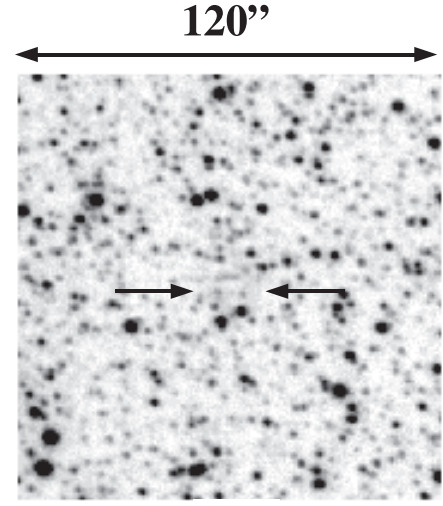

87

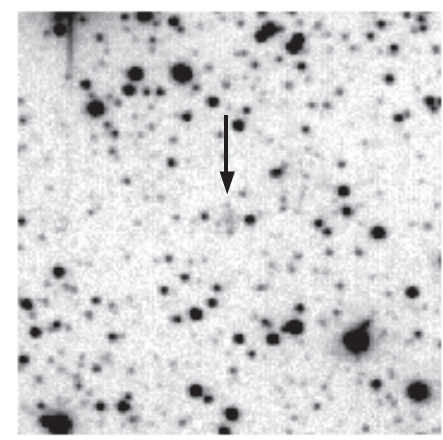

90

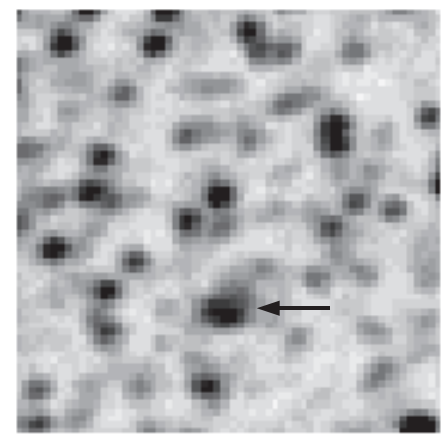

93

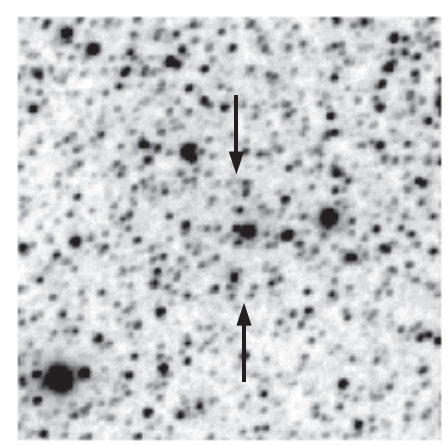

96

Fig. A.8. As for Fig. A.1, but showing the finding charts for PN 85-96. [SIII] images are shown for PN 91, 93, and 94. 


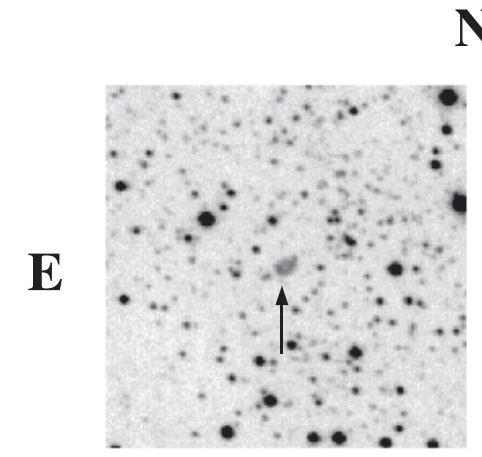

97

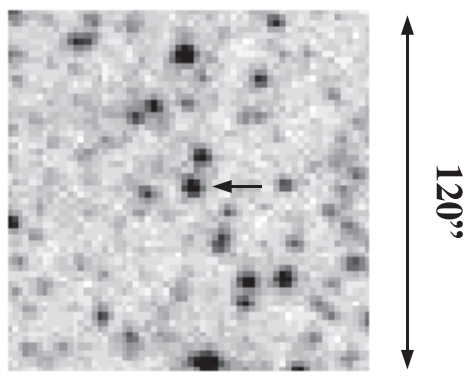

98

Fig. A.9. As for Fig. A.1, but showing the finding charts for PN 97-98. [SIII] images are shown for PN 98.

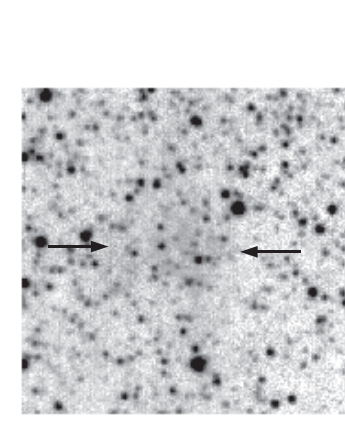

1

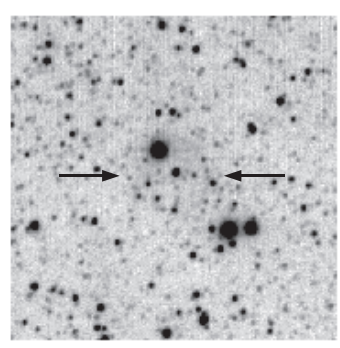

$\mathbf{E}$

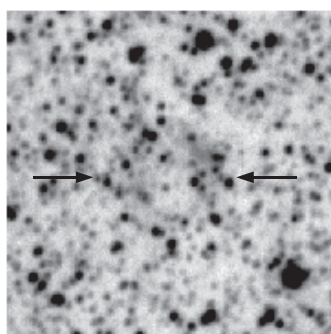

7

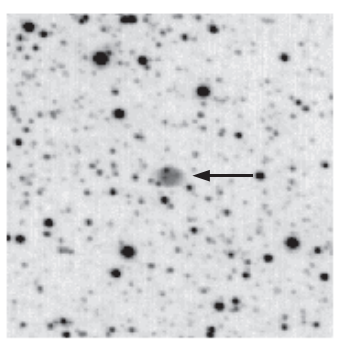

10

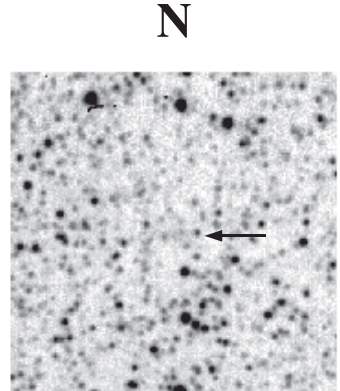

2

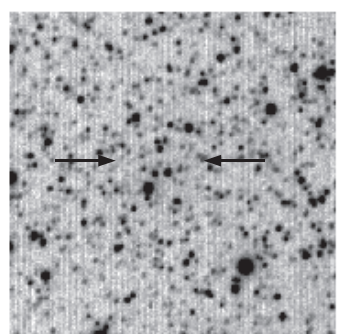

5

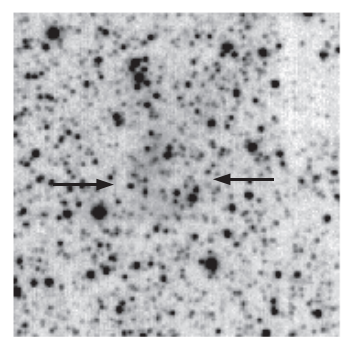

8

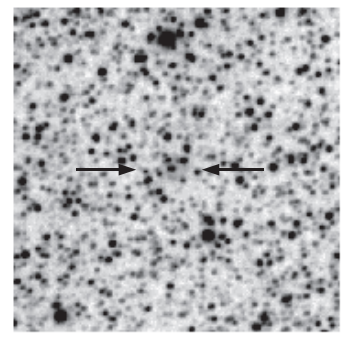

11

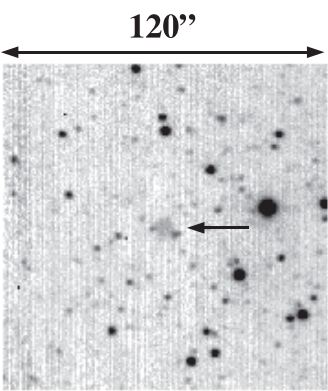

3

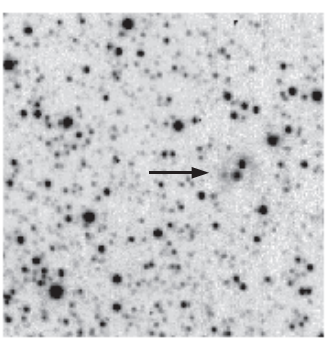

6

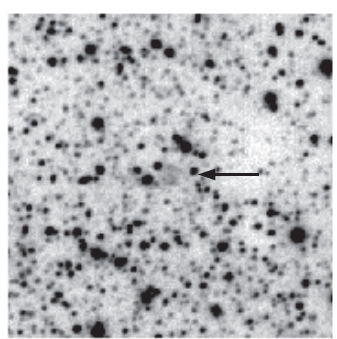

9

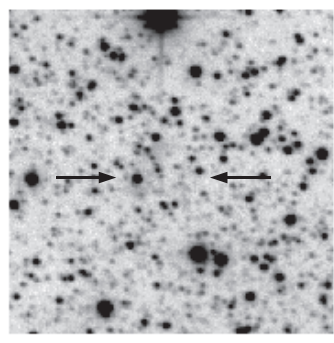

12

Fig. A.10. Finding charts for the PN candidates discovered in the follow-up $\mathrm{H} \alpha$ survey identified as number 1-12. Otherwise, these are similar to Fig. A.1. 


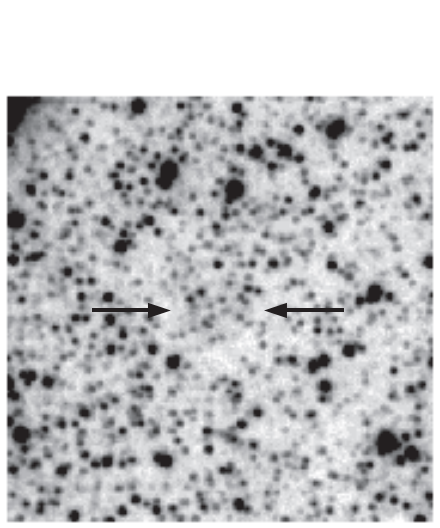

13

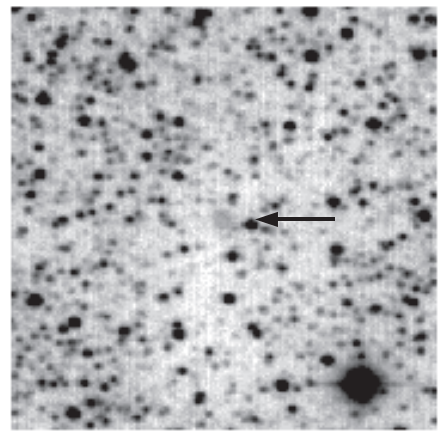

$\mathbf{E}$

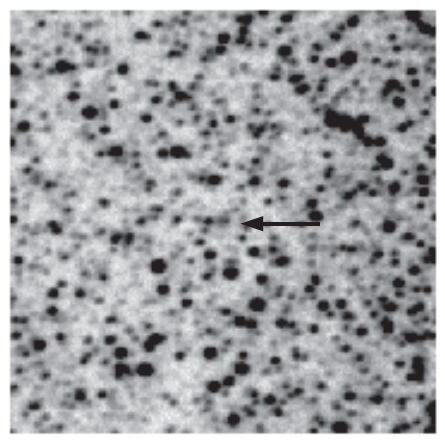

19

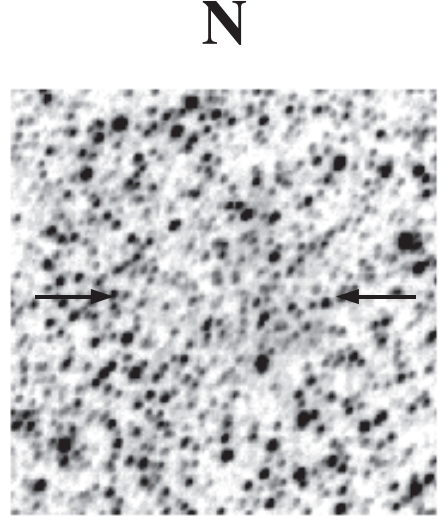

14

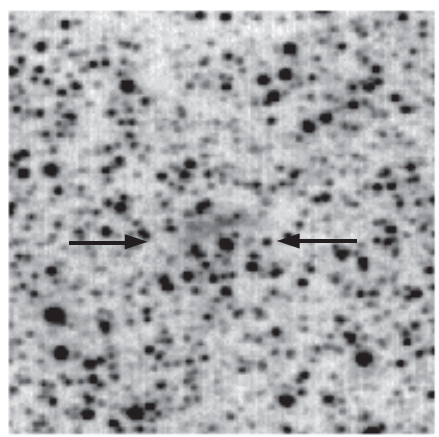

17

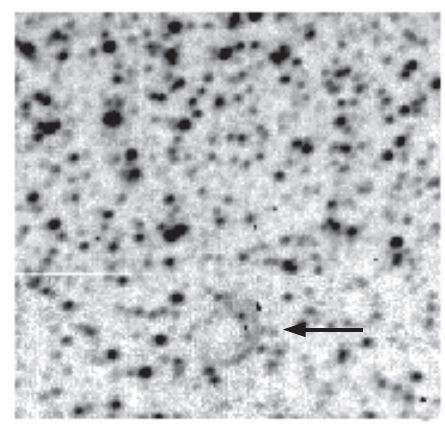

20

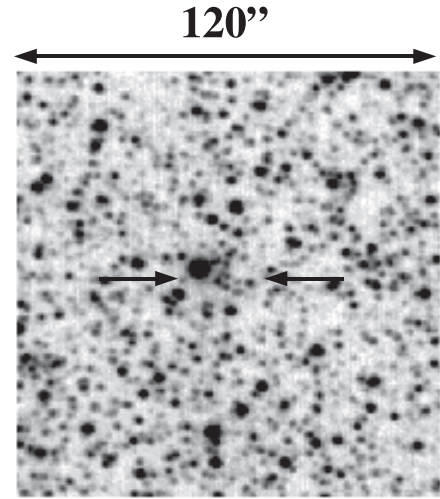

15

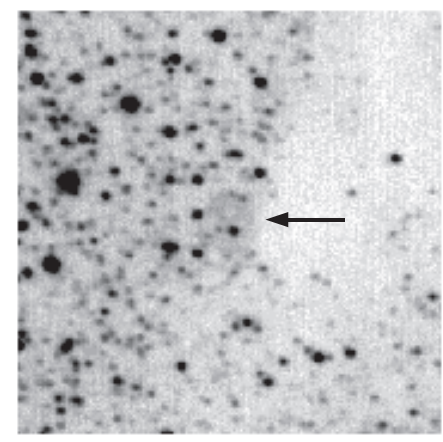

18

Fig. A.11. As for Fig. A.10, but for PN 13-20. 


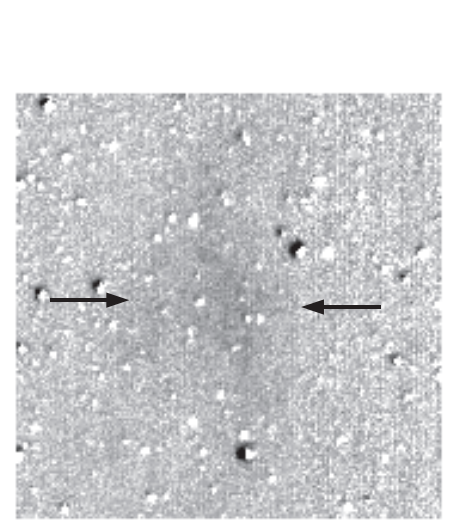

1

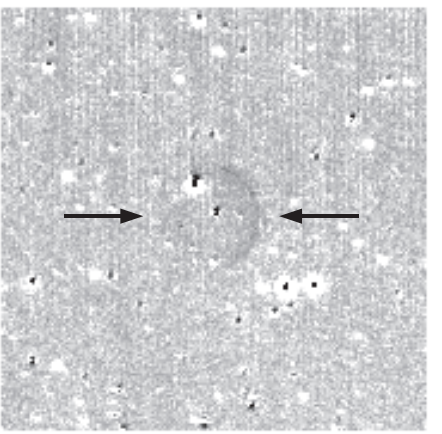

$\mathbf{E}$

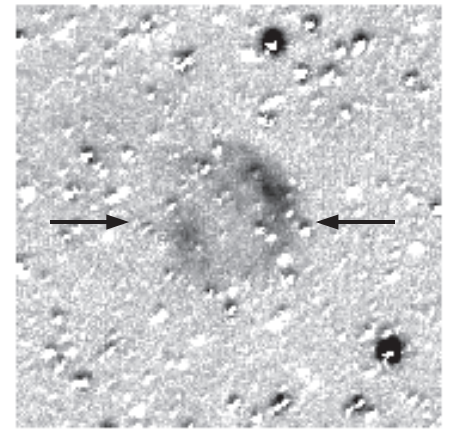

7

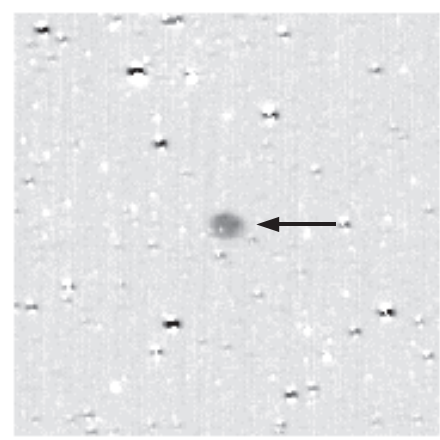

10

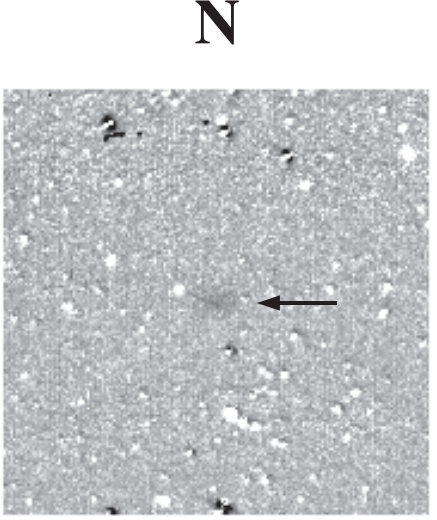

2

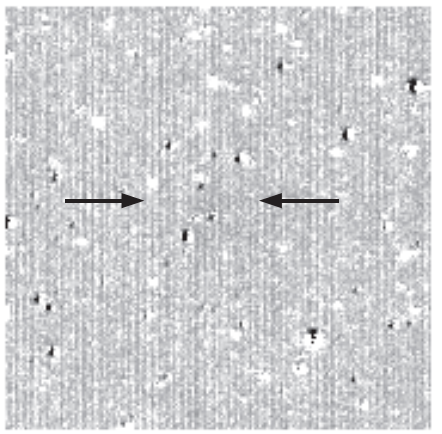

5

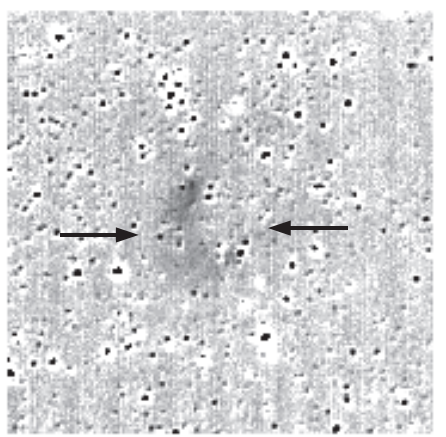

8

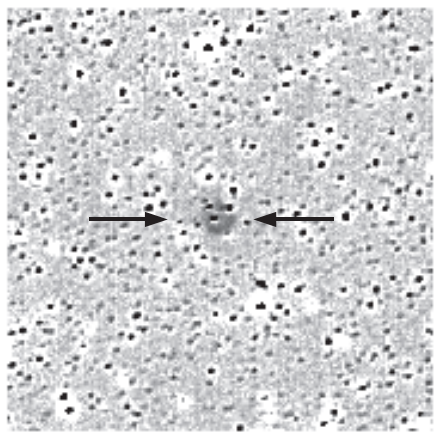

11

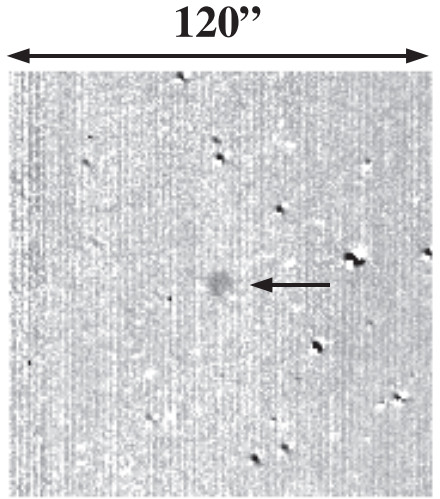

3

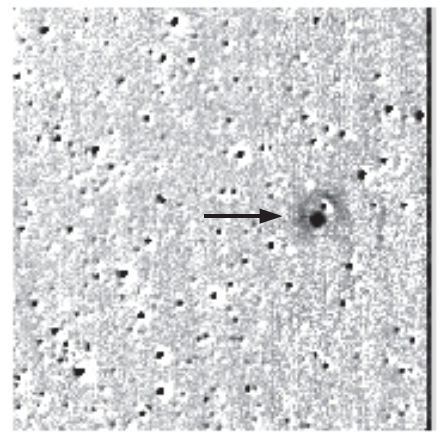

6

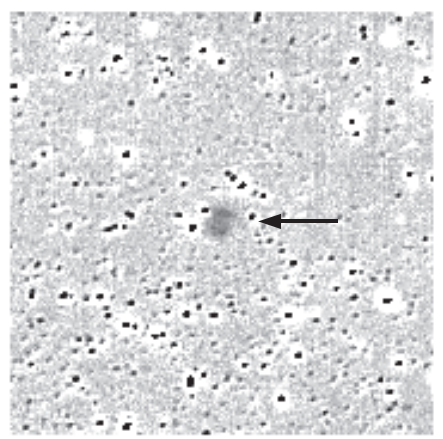

9

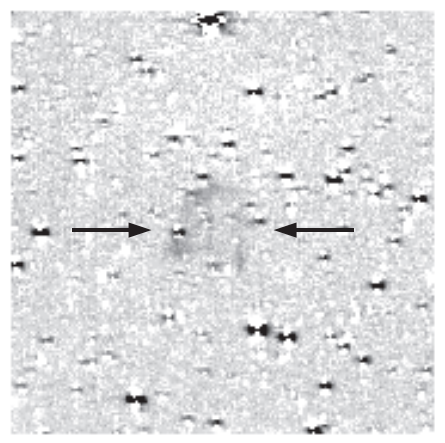

12

Fig. A.12. As for Fig. A.10, again showing candidates 1-12, but now with the continuum image subtracted to illustrate better these very low surface brightness PNe. 


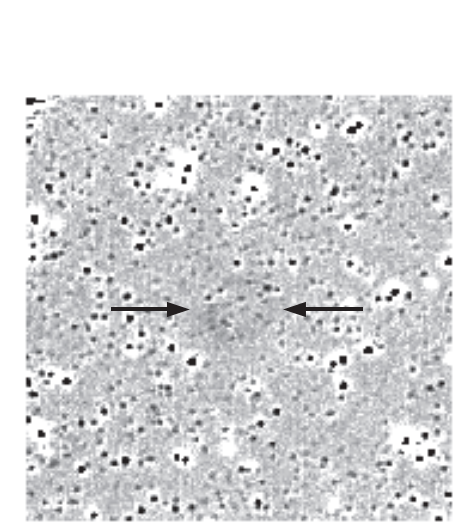

13

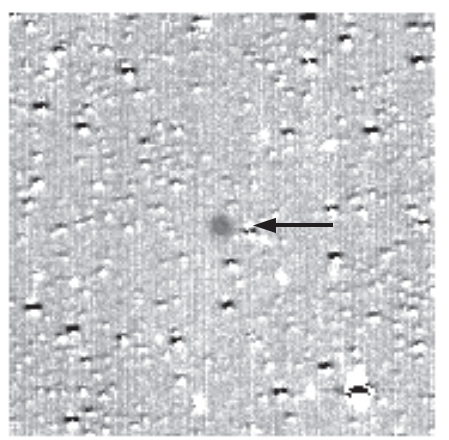

$\mathbf{E}$

16

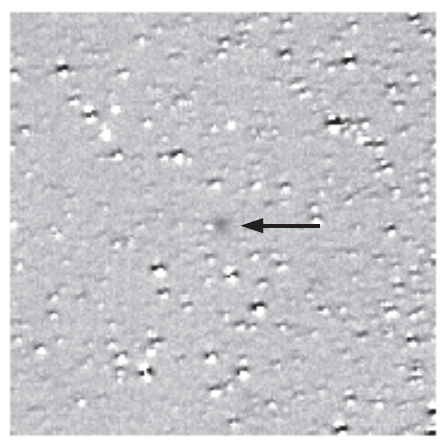

19

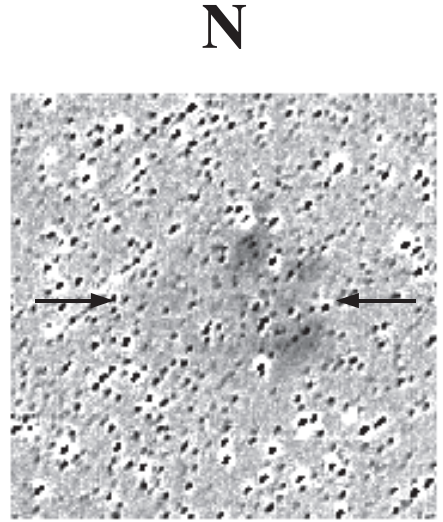

14

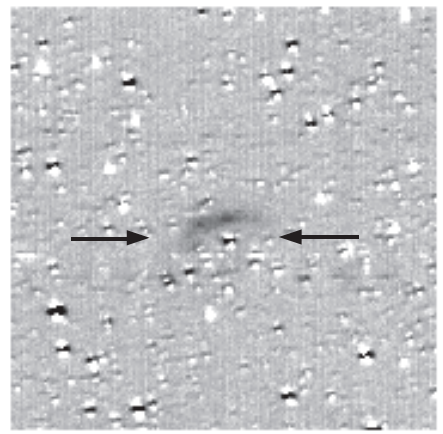

17

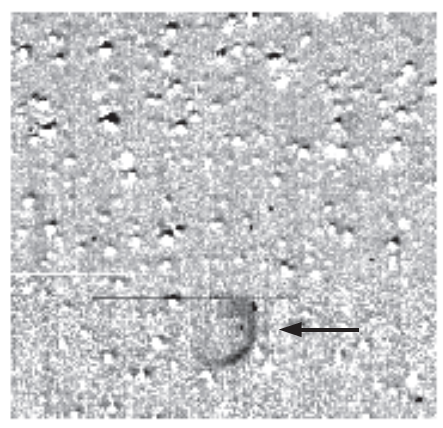

20

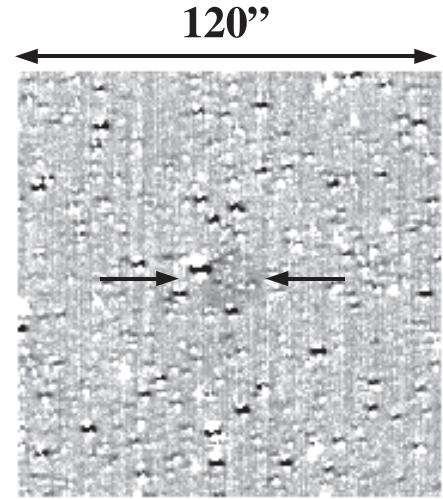

15

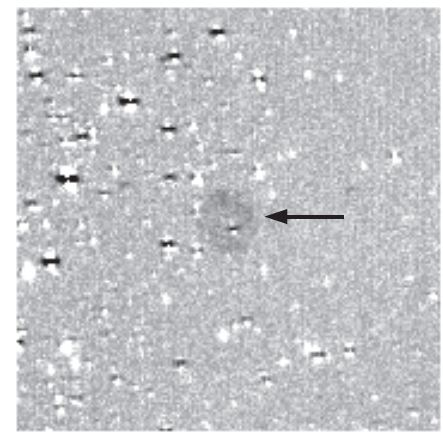

18

Fig. A.13. As for Fig. A.12, but for PN 13-20. 


\section{References}

Beaulieu, S. F., Dopita, M. A., \& Freeman, K. C. 1999, ApJ, 515, 610 Boumis, P., Paleologou, E. V., Mavromatakis, F., \& Papamastorakis, J. 2003, MNRAS, 339, 735

Ciardullo, R. 1995, IAU Highlights of Astronomy, ed. I. Appenzeller (Dordrecht: Kluwer), 10, 507

Dopita, M. A., Vassiliadis, E., Wood, P. R., et al. 1997, ApJ, 474, 188

Dwek, E., Arendt, R. G., Hauser, M. G., et al. 1995, ApJ, 445, 716

Eisenhauer, F., Schödel, R., Genzel, R., et al. 2003, ApJ, 597, L121

Forestini, M., \& Charbonnel, C. 1997, A\&AS, 123, 241

Gómez, Y., Rodrigues, L. F., \& Mirabel, I. F. 1997, Rev. Mex. Astr. Ap., 33, 25

Hui, X., Ford, H. C., Freeman, K. C., \& Dopita, M. A. 1995, ApJ, 449, 592

Kohoutek, L. 1994, Astron. Nachr., 315, 235

Kohoutek, L. 2002, Astron. Nachr., 323, 57

McWilliam, A., \& Rich, M. R. 1994, ApJS, 91, 749
Muller, G. P., Reed, R., Armandroff, T., Boroson, T., \& Jacoby, G., Proc. SPIE, 3355, 487

Parker, Q. A., et al. 2003, Planetary Nebulae: Their Evolution and Role in the Universe, ed. S. Kwok, M. Dopita, \& P. Sutherland (San Francisco: ASP), IAU Symp., 209, 25

Parker, Q. A., \& Phillipps, S. 1998, PASA, 15, 28

Peimbert, M. 1987, Rev. Mex. Astr. Ap., 14, 166

Peimbert, M. 1993, in Planetary Nebulae, IAU Symp., 155, 523

Pottasch, S. R., Bignell, C., Olling, R., \& Zijlstra, A. A. 1988, A\&A, 205, 248

Sevenster, M., Chapman, J. M., Habing, H. J., Killeen, N. E. B., \& Lindqvist, M. 1997, A\&A, 122, 79

Valdes, F. G. 1998, in Astronomical Data Analysis Software and Systems VII, Astr. Soc. Pac. Conf. Ser., 145, 53

Van de Steene, G. C., \& Jacoby, G. H. 2001, A\&A, 373, 536

Van de Steene, G. C., \& Pottasch, S. R. 1995, A\&A, 299, 238 